\title{
Capacity Results for Block-Stationary Gaussian Fading Channels with a Peak Power Constraint
}

\author{
Jun Chen and Venugopal V. Veeravalli \\ ECE Department \& Coordinated Science Lab \\ University of Illinois at Urbana-Champaign \\ Urbana, IL 61801, USA \\ Email: junchen,vvv@uiuc.edu
}

\begin{abstract}
We consider a peak-power-limited single-antenna block-stationary Gaussian fading channel where neither the transmitter nor the receiver knows the channel state information, but both know the channel statistics. This model subsumes most previously studied Gaussian fading models. We first compute the asymptotic channel capacity in the high SNR regime and show that the behavior of channel capacity depends critically on the channel model. For the special case where the fading process is symbol-by-symbol stationary, we also reveal a fundamental interplay between the codeword length, communication rate, and decoding error probability. Specifically, we show that the codeword length must scale with SNR in order to guarantee that the communication rate can grow logarithmically with SNR with bounded decoding error probability, and we find a necessary condition for the growth rate of the codeword length. We also derive an expression for the capacity per unit energy. Furthermore, we show that the capacity per unit energy is achievable using temporal ON-OFF signaling with optimally allocated ON symbols, where the optimal ON-symbol allocation scheme may depend on the peak power constraint.
\end{abstract}

Index Terms

Wireless channels, Noncoherent capacity, Capacity per unit cost, Block fading

\section{INTRODUCTION}

The capacity analysis of noncoherent fading channels has received considerable attention in recent years since it provides the ultimate limit on the rate of reliable communication on such channels.

Proposed approaches to modeling noncoherent fading channels can be classified into two broad categories. The first is to model the fading process as a block-independent process. In the standard version of this model [1], the channel remains constant over blocks consisting of $T$ symbol periods, and changes independently from block to block. The second is to model the fading process as a symbol-by-symbol stationary process. In this model, the independence assumption is removed, but the block structure is not allowed. Somewhat surprisingly, these two models lead to very different capacity results. For the standard block fading model, the capacity is shown [1], [2] to 
grow logarithmically with SNR, while for the symbol-by-symbol stationary model, the capacity grows only doublelogarithmically in SNR at high SNR if the fading process is regular [3]-[5]. For symbol-by-symbol stationary Gaussian fading channels, if the Lebesgue measure of the set of harmonics where the spectral density of the fading process is zero is positive, the fading process is nonregular and the capacity grows logarithmically with SNR [6]. This result is consistent with the capacity result for block-independent fading channels in the sense that the $\log$ SNR behavior in the high SNR regime results from the rank deficiency of the correlation matrix of the fading process. This point was elucidated in [7] where a time-selective block fading model was considered in which the rank of the correlation within the block is allowed to be any number between one and the blocklength.

However, the mechanisms that cause the rank deficiency in the block-independent fading and nonregular symbolby-symbol stationary models are different. For the block-independent fading model, the rank deficiency happens within each block. But for the nonregular symbol-by-symbol stationary fading channel model, the correlation matrix of the fading process over any finite block can still be full-rank; the rank deficiency in this case is in the asymptotic sense. In general, the rank deficiency of the correlation matrix can be affected by both the short timescale correlation of the fading process as in the block-independent fading model and large timescale correlation as in the symbolby-symbol stationary channel model. In order to capture both of these effects, we model the fading process as a block-stationary Gaussian process.

The block-stationary model was introduced and justified in [7]. We summarize the main points of the justification here. In the block-independent fading model, the channel is assumed to change in an i.i.d. manner from block to block. The independence can be justified in certain time division or frequency hopping systems where the blocks are separated sufficiently in time or frequency to undergo independent fading. The independence assumption is also convenient for information-theoretic analysis as it allows us to focus on one block in studying the capacity. If the blocks are not separated far enough in time or frequency, the fading process can be correlated across blocks and the block-stationary model is more appropriate in this scenario. Without time or frequency hopping, the channel variations from one block to the next are dictated by the long term variations in the scattering environment. If we assume that the variations in average channel power are compensated for by other means such as power control, it is reasonable to model the variation from block to block as stationary and ergodic.

Remark 1: The block-stationary model does not imply that the fading process is stationary on a symbol-bysymbol basis as in the analysis of [3], [6]. But as explained in [7], the symbol-by-symbol stationary model is not realistic for time intervals that are larger than that corresponding to a few wavelengths. For this reason it may be more accurate to model the fading process using a block fading model with possible correlation across blocks than it is to model it as a symbol-by-symbol stationary process. From the viewpoint of analysis, the block-stationary model generalizes all previously considered models discussed above and therefore so do the capacity results for this model. More importantly, the block-stationary model provides us with a framework to study the interplay between many aspects of fading channels which are not captured in the aforementioned models, and allows us to identify the properties that are shared by different models and the properties that depend on channel modelling.

The channel capacity for the block-stationary model was only studied in [7] under certain constraints on the 
correlation structure across blocks, which essentially disallow rank deficiency over the large timescale. In this paper, we conduct a more complete study of the capacity for this channel model.

\section{Notation And System Model}

\section{A. Notation}

The following notation is used in paper. For deterministic objects, uppercase letters denote matrices, lowercase letters denote scalars, and underlined lowercase letters denote vectors. Random objects are identified by corresponding boldfaced letters. For example, $\boldsymbol{X}$ denotes a random matrix, $X$ denotes the realization of $\boldsymbol{X}, \underline{\boldsymbol{x}}$ denotes a random vector, and $\boldsymbol{x}$ denotes a random scalar. For simplicity, sometimes we also use $\boldsymbol{x}^{n}$ to denote the random vector $\left(\boldsymbol{x}_{1}, \boldsymbol{x}_{2}, \cdots, \boldsymbol{x}_{n}\right)^{\top}$. Although uppercase letters are typically used for matrices, there are some exceptions, and these exceptions are noted explicitly in the paper. The operators det, $\operatorname{tr}, *, \top$, and $\dagger$ denote determinant, trace, conjugate, transpose and conjugate transpose, respectively. We let $I_{M}$ denote the $M \times M$ identity matrix for any positive integer $M$, and let $\operatorname{var}(\underline{\boldsymbol{a}} \mid \underline{\boldsymbol{b}})$ denote $\mathbb{E}\left[(\underline{\boldsymbol{a}}-\mathbb{E}(\underline{\boldsymbol{a}} \mid \underline{\boldsymbol{b}}))(\underline{\boldsymbol{a}}-\mathbb{E}(\underline{\boldsymbol{a}} \mid \underline{\boldsymbol{b}}))^{\dagger}\right]$ for random vectors $\underline{\boldsymbol{a}}$ and $\underline{\boldsymbol{b}}$.

\section{B. System Model}

We consider a discrete-time channel whose time- $t$ complex-valued output $\boldsymbol{y}_{t} \in \mathbb{C}$ is given by

$$
\boldsymbol{y}_{t}=\boldsymbol{h}_{t} \boldsymbol{x}_{t}+\boldsymbol{z}_{t}
$$

where $\boldsymbol{x}_{t} \in \mathbb{C}$ is the input at time $t$ with peak power constraint $\left|\boldsymbol{x}_{t}\right|^{2} \leq \mathrm{SNR} ;\left\{\boldsymbol{h}_{t}\right\}$ models the fading process; and $\left\{\boldsymbol{z}_{t}\right\}$ models additive noise. The processes $\left\{\boldsymbol{h}_{t}\right\}$ and $\left\{\boldsymbol{z}_{t}\right\}$ are assumed to be independent and have a joint distribution that does not depend on the input $\left\{\boldsymbol{x}_{t}\right\}$. We assume that $\left\{\boldsymbol{z}_{t}\right\}$ is a sequence of i.i.d. circularly symmetric complex-Gaussian random variables of zero mean and unit variance, i.e., $\boldsymbol{z}_{t} \sim \mathcal{C N}(0,1)$. We assume that the fading process $\left\{\boldsymbol{h}_{t}\right\}$ is a block-stationary process with $\boldsymbol{h}_{t} \sim \mathcal{C N}(0,1)$ and block length $T$, i.e., $\left\{\underline{\boldsymbol{h}}_{k}=\right.$ $\left.\left(\boldsymbol{h}_{k T+1}, \boldsymbol{h}_{k T+2}, \cdots, \boldsymbol{h}_{k T+T}\right)^{\top}\right\}_{k}$ is a vector-valued stationary process. Furthermore, we assume that $\left\{\underline{\boldsymbol{h}}_{k}\right\}$ is an ergodic process with a matrix spectral density function $S\left(e^{j \omega}\right),-\pi \leq \omega \leq \pi$. Specifically,

$$
S\left(e^{j \omega}\right)=\sum_{i=-\infty}^{\infty} R(i) e^{-j \omega i}
$$

where $R(i)=\mathbb{E} \underline{\boldsymbol{h}}_{k} \underline{\boldsymbol{h}}_{k-i}^{\dagger}$. Since $R(i)=R^{\dagger}(-i), i \in \mathbb{Z}$, it is not hard to check that $S\left(e^{j \omega}\right)$ is Hermitian, i.e., $S\left(e^{j \omega}\right)=S^{\dagger}\left(e^{j \omega}\right)$. Moreover, we have $S\left(e^{j \omega}\right) \succcurlyeq 0(-\pi \leq \omega \leq \pi)$, i.e., $S\left(e^{j \omega}\right)$ is a positive semi-definite matrix.

There is an interesting relation between the matrix spectral density function and the asymptotic prediction error. Specifically, for the block stationary process $\left\{\boldsymbol{h}_{t}+\frac{1}{\sqrt{\mathrm{SNR}}} \boldsymbol{z}_{t}\right\}$, define the following prediction error covariance matrices:

$$
\begin{aligned}
\Sigma(\mathrm{SNR}) & \triangleq \operatorname{var}\left(\left(\boldsymbol{h}_{1}+\frac{1}{\sqrt{\mathrm{SNR}}} \boldsymbol{z}_{1}, \boldsymbol{h}_{2}+\frac{1}{\sqrt{\mathrm{SNR}}} \boldsymbol{z}_{2}, \cdots, \boldsymbol{h}_{T}+\frac{1}{\sqrt{\mathrm{SNR}}} \boldsymbol{z}_{T}\right)^{\top} \mid\left\{\boldsymbol{h}_{t}+\frac{1}{\sqrt{\mathrm{SNR}}} \boldsymbol{z}_{t}\right\}_{t=-\infty}^{0}\right) \\
\Sigma(\infty) & \triangleq \operatorname{var}\left(\left(\boldsymbol{h}_{1}, \boldsymbol{h}_{2}, \cdots, \boldsymbol{h}_{T}\right)^{\top} \mid\left\{\boldsymbol{h}_{t}\right\}_{t=-\infty}^{0}\right) .
\end{aligned}
$$


Then $\Sigma(\mathrm{SNR})$ and $\Sigma(\infty)$ are related to the matrix spectral density function $S\left(e^{j \omega}\right)$ of $\left\{\boldsymbol{h}_{t}\right\}$ as [8]

$$
\begin{aligned}
\operatorname{det}[\Sigma(\mathrm{SNR})] & =\exp \left\{\frac{1}{2 \pi} \int_{-\pi}^{\pi} \log \operatorname{det}\left[S\left(e^{j \omega}\right)+\frac{1}{\mathrm{SNR}} I_{T}\right] \mathrm{d} \omega\right\}, \\
\operatorname{det}[\Sigma(\infty)] & =\exp \left\{\frac{1}{2 \pi} \int_{-\pi}^{\pi} \log \operatorname{det}\left[S\left(e^{j \omega}\right)\right] \mathrm{d} \omega\right\} .
\end{aligned}
$$

The remainder of this paper is organized as follows. In Section [II] we establish single-letter upper and lower bounds on channel capacity, and use these bounds to analyze the asymptotic capacity in the high SNR regime. In Section IV we discuss the robustness of the asymptotic capacity results, and the interplay between the codeword length, communication rate and decoding error probability. In Section $\nabla$ we adapt the formula of Verdú for capacity per unit cost [9] to our channel model, and use it to derive an expression for the capacity per unit energy in the presence of a peak power constraint. We summarize our results in Section [V]

\section{ASYMPtotic CAPACITY AT High SNR}

We denote the capacity with peak power constraint SNR by $C(\mathrm{SNR})$. For any $n \in \mathbb{N}$ and SNR $>0$, let

$$
\mathbb{D}_{n}(\mathrm{SNR})=\left\{x^{n} \in \mathbb{C}^{n}: \max _{1 \leq t \leq n}\left|x_{t}\right|^{2} \leq \mathrm{SNR}\right\} .
$$

Let $\mathcal{P}_{n}(\mathrm{SNR})$ be the set of probability distributions on $\mathbb{D}_{n}(\mathrm{SNR})$. Since the channel is block-wise stationary and ergodic, a coding theorem exists and we have

$$
C(\mathrm{SNR})=\lim _{n \rightarrow \infty} \sup _{P_{\boldsymbol{x}^{n}} \in \mathcal{P}_{n}(\mathrm{SNR})} \frac{1}{n} I\left(\boldsymbol{x}^{n} ; \boldsymbol{y}^{n}\right) .
$$

\section{A. Lower Bound and Upper Bound}

To derive a lower bound on $C(\mathrm{SNR})$ for the channel model given in 11, we adopt the interleaved decisionoriented training scheme proposed in [10] with some modifications. This scheme can also be viewed as a way of interpreting the computations in $[3$, Sec. IV.E], $[6$, Sec. V].

Let $p(\boldsymbol{x})$ be a circularly symmetric distribution with $\|\boldsymbol{x}\|^{2} \in\left[x_{\text {min }}^{2}, \mathrm{SNR}\right]$. Construct the codebook $\mathcal{C}=\mathcal{C}_{1} \times \mathcal{C}_{2} \times$ $\cdots \times \mathcal{C}_{K}$ with $K$ sub-codebooks $\mathcal{C}_{1}, \mathcal{C}_{2}, \cdots, \mathcal{C}_{K}$, where codebook $\mathcal{C}_{i}(i=1, \cdots, K)$ contains $2^{n R_{i}}$ codewords of length $n$ generated independently symbol by symbol using distribution $p(\boldsymbol{x})$. We assume that $K$ is a multiple of the block length $T$, i.e., $K=r T$ for some positive integer $r$.

Now we multiplex (or interleave) these $K$ codebooks. Specifically, codebook $\mathcal{C}_{i}(i=1, \cdots, K)$ is used at time instants $i, i+K, i+2 K, \cdots, i+(n-1) K$. For codebook $\mathcal{C}_{i}$, its codeword can be successfully decoded if

$$
R_{1} \leq \frac{1}{n} I\left(\left\{\boldsymbol{x}_{1+j K}\right\}_{j=0}^{n-1} ;\left\{\boldsymbol{y}_{1+j K}\right\}_{j=0}^{n-1}\right)
$$

for sufficiently large $n$. Furthermore, using the facts that $\left\{\boldsymbol{x}_{1+j K}\right\}_{j=0}^{n-1}$ are i.i.d. and that the channel is stationary 
over time instants $1,1+K, 1+2 K, \cdots, 1+(n-1) K$, we get

$$
\begin{aligned}
\frac{1}{n} I\left(\left\{\boldsymbol{x}_{1+j K}\right\}_{j=0}^{n-1} ;\left\{\boldsymbol{y}_{1+j K}\right\}_{j=0}^{n-1}\right) & =\frac{1}{n} \sum_{j=0}^{n-1} I\left(\boldsymbol{x}_{1+j K} ;\left\{\boldsymbol{y}_{1+i K}\right\}_{i=0}^{n-1} \mid\left\{\boldsymbol{x}_{1+i K}\right\}_{i=j+1}^{n-1}\right) \\
& =\frac{1}{n} \sum_{j=0}^{n-1} I\left(\boldsymbol{x}_{1+j K} ;\left\{\boldsymbol{y}_{1+i K}\right\}_{i=0}^{n-1},\left\{\boldsymbol{x}_{1+i K}\right\}_{i=j+1}^{n-1}\right) \\
& \geq \frac{1}{n} \sum_{j=0}^{n-1} I\left(\boldsymbol{x}_{1+j K} ; \boldsymbol{y}_{1+j K}\right) \\
& =I\left(\boldsymbol{x}_{1} ; \boldsymbol{y}_{1}\right)
\end{aligned}
$$

This is to be expected since a channel with memory has a higher reliable communication rate than the memoryless channel with the same marginal transition probability. Thus, reliable communication at rate $R_{1}=I\left(\boldsymbol{x}_{1} ; \boldsymbol{y}_{1}\right)$ is possible for sub-codebook $\mathcal{C}_{1}$. After $\left\{\boldsymbol{x}_{1+j K}\right\}_{j=0}^{n-1}$ is successfully decoded, the receiver can use these values as well as $\left\{\boldsymbol{y}_{1+j K}\right\}_{j=0}^{n-1}$ to estimate $\left\{\boldsymbol{h}_{2+j K}\right\}_{j=0}^{n-1}$. Specifically, $\left(\boldsymbol{x}_{1+j K}, \boldsymbol{y}_{1+j K}\right)$ is used to estimate $\boldsymbol{h}_{2+j K}, j=0,1, \cdots, n-1$. Since $\|\boldsymbol{x}\|^{2} \in\left[x_{\min }^{2}, \mathrm{SNR}\right]$, it is easy to verify the following Markov chain condition

$$
\boldsymbol{h}_{1+j K}+\frac{1}{x_{\min }} \boldsymbol{z}_{1+j K} \rightarrow\left(\boldsymbol{x}_{1+j K}, \boldsymbol{y}_{1+j K}\right) \rightarrow \boldsymbol{h}_{2+j K} .
$$

To facilitate the calculation, we assume that $\boldsymbol{h}_{1+j K}+\frac{1}{x_{\min }} \boldsymbol{z}_{1+j K}$ is used to estimate $\boldsymbol{h}_{2+j K}$ by forming the MMSE estimate $\mathbb{E}\left(\boldsymbol{h}_{2+j K} \mid \boldsymbol{h}_{1+j K}+\frac{1}{x_{\min }} \boldsymbol{z}_{1+j K}\right), j=0,1, \cdots, n-1$. The receiver decodes the codeword in codebook $\mathcal{C}_{2}$ using $\left\{\mathbb{E}\left(\boldsymbol{h}_{2+j K} \mid \boldsymbol{h}_{1+j K}+\frac{1}{x_{\min }} \boldsymbol{z}_{1+j K}\right)\right\}_{j=0}^{n-1}$ as side information. Successful decoding is possible if

$$
R_{2} \leq \frac{1}{n} \sum_{j=0}^{n-1} I\left(\left\{\boldsymbol{x}_{2+j K}\right\}_{j=0}^{n-1} ;\left\{\boldsymbol{y}_{2+j K}\right\}_{j=0}^{n-1},\left\{\mathbb{E}\left(\boldsymbol{h}_{2+j K} \mid \boldsymbol{h}_{1+j K}+\frac{1}{x_{\min }} \boldsymbol{z}_{1+j K}\right)\right\}_{j=0}^{n-1}\right) .
$$

Similar to (4), we can use the lower bound

$$
\begin{aligned}
& \frac{1}{n} \sum_{j=0}^{n-1} I\left(\left\{\boldsymbol{x}_{2+j K}\right\}_{j=0}^{n-1} ;\left\{\boldsymbol{y}_{2+j K}\right\}_{j=0}^{n-1},\left\{\mathbb{E}\left(\boldsymbol{h}_{2+j K} \mid \boldsymbol{h}_{1+j K}+\frac{1}{x_{\min }} \boldsymbol{z}_{1+j K}\right)\right\}_{j=0}^{n-1}\right) \\
\geq & I\left(\boldsymbol{x}_{2} ; \boldsymbol{y}_{2}, \mathbb{E}\left(\boldsymbol{h}_{2} \mid \boldsymbol{h}_{1}+\frac{1}{x_{\min }} \boldsymbol{z}_{1}\right)\right) \\
= & I\left(\boldsymbol{x}_{2} ; \boldsymbol{y}_{2} \mid \mathbb{E}\left(\boldsymbol{h}_{2} \mid \boldsymbol{h}_{1}+\frac{1}{x_{\min }} \boldsymbol{z}_{1}\right)\right)
\end{aligned}
$$

to show that reliable communication at rate $R_{2}=I\left(\boldsymbol{x}_{2} ; \boldsymbol{y}_{2} \mid \mathbb{E}\left(\boldsymbol{h}_{2} \mid \boldsymbol{h}_{1}+\frac{1}{x_{\min }} \boldsymbol{z}_{1}\right)\right)$ is possible for sub-codebook $\mathcal{C}_{2}$. By applying this procedure successively, we can conclude that for codebook $\mathcal{C}_{i}$, reliable communication is possible at rate

$$
R_{i}=I\left(\boldsymbol{x}_{i} ; \boldsymbol{y}_{i} \mid \mathbb{E}\left(\boldsymbol{h}_{i} \mid\left\{\boldsymbol{h}_{j}+\frac{1}{x_{\min }} \boldsymbol{z}_{j}\right\}_{j=1}^{i-1}\right)\right), \quad i=1,2, \cdots, K .
$$

Thus, using this interleaved decision-oriented training scheme, we can have reliable communication at overall rate of

$$
R=\frac{1}{K} \sum_{i=1}^{K} R_{i}=\frac{1}{K} \sum_{i=1}^{K} I\left(\boldsymbol{x}_{i} ; \boldsymbol{y}_{i} \mid \mathbb{E}\left(\boldsymbol{h}_{i} \mid\left\{\boldsymbol{h}_{j}+\frac{1}{x_{\min }} \boldsymbol{z}_{j}\right\}_{j=1}^{i-1}\right)\right)
$$


We show in Appendix 凹 that $\left\{I\left(\boldsymbol{x}_{i+j T} ; \boldsymbol{y}_{i+j T} \mid \mathbb{E}\left(\boldsymbol{h}_{i+j T} \mid\left\{\boldsymbol{h}_{k}+\frac{1}{x_{\min }} \boldsymbol{z}_{k}\right\}_{k=1}^{i+j T-1}\right)\right)\right\}_{j}$ is a monotone increasing sequence with

$\lim _{j \rightarrow \infty} I\left(\boldsymbol{x}_{i+j T} ; \boldsymbol{y}_{i+j T} \mid \mathbb{E}\left(\boldsymbol{h}_{i+j T} \mid\left\{\boldsymbol{h}_{k}+\frac{1}{x_{\min }} \boldsymbol{z}_{k}\right\}_{k=1}^{i+j T-1}\right)\right)=I\left(\boldsymbol{x}_{i} ; \boldsymbol{y}_{i} \mid \mathbb{E}\left(\boldsymbol{h}_{i} \mid\left\{\boldsymbol{h}_{k}+\frac{1}{x_{\min }} \boldsymbol{z}_{k}\right\}_{k=-\infty}^{i-1}\right)\right)$.

Now we let $K$ go to infinity (i.e., we let $r \rightarrow \infty$ since $T$ is fixed), and we obtain

$$
\begin{aligned}
\lim _{K \rightarrow \infty} R & =\lim _{K \rightarrow \infty} \frac{1}{K} \sum_{i=1}^{K} I\left(\boldsymbol{x}_{i} ; \boldsymbol{y}_{i} \mid \mathbb{E}\left(\boldsymbol{h}_{i} \mid\left\{\boldsymbol{h}_{j}+\frac{1}{x_{\min }} \boldsymbol{z}_{j}\right\}_{j=1}^{i-1}\right)\right) \\
& =\lim _{K \rightarrow \infty} \frac{1}{K} \sum_{i=1}^{T} \sum_{j=0}^{r-1} I\left(\boldsymbol{x}_{i+j T} ; \boldsymbol{y}_{i+j T} \mid \mathbb{E}\left(\boldsymbol{h}_{i+j T} \mid\left\{\boldsymbol{h}_{k}+\frac{1}{x_{\min }} \boldsymbol{z}_{k}\right\}_{k=1}^{i+j T-1}\right)\right) \\
& =\frac{1}{T} \sum_{i=1}^{T}\left[\lim _{r \rightarrow \infty} \frac{1}{r} \sum_{j=0}^{r-1} I\left(\boldsymbol{x}_{i+j T} ; \boldsymbol{y}_{i+j T} \mid \mathbb{E}\left(\boldsymbol{h}_{i+j T} \mid\left\{\boldsymbol{h}_{k}+\frac{1}{x_{\min }} \boldsymbol{z}_{k}\right\}_{k=1}^{i+j T-1}\right)\right)\right] \\
& =\frac{1}{T} \sum_{i=1}^{T} I\left(\boldsymbol{x}_{i} ; \boldsymbol{y}_{i} \mid \mathbb{E}\left(\boldsymbol{h}_{i} \mid\left\{\boldsymbol{h}_{k}+\frac{1}{x_{\min }} \boldsymbol{z}_{k}\right\}_{k=-\infty}^{i-1}\right)\right) \\
& =\frac{1}{T} \sum_{i=1}^{T} I\left(\boldsymbol{x}_{i} ; \boldsymbol{h}_{i} \boldsymbol{x}_{i}+\boldsymbol{z}_{i} \mid \mathbb{E}\left(\boldsymbol{h}_{i} \mid\left\{\boldsymbol{h}_{k}+\frac{1}{x_{\min }} \boldsymbol{z}_{k}\right\}_{k=-\infty}^{i-1}\right)\right) .
\end{aligned}
$$

This yields the single-letter lower bound

$$
C(\mathrm{SNR}) \geq \frac{1}{T} \sum_{i=1}^{T} I\left(\boldsymbol{x}_{i} ; \boldsymbol{h}_{i} \boldsymbol{x}_{i}+\boldsymbol{z}_{i} \mid \mathbb{E}\left(\boldsymbol{h}_{i} \mid\left\{\boldsymbol{h}_{k}+\frac{1}{x_{\min }} \boldsymbol{z}_{k}\right\}_{k=-\infty}^{i-1}\right)\right)
$$

where $\boldsymbol{x}_{1}, \boldsymbol{x}_{2}, \cdots, \boldsymbol{x}_{T}$ all have the same distribution $p(\boldsymbol{x})$, which is to be optimized later.

Remark 2: Although channel estimation and communication are intertwined in this interleaved decision-oriented training scheme, the effect of channel memory is isolated from channel coding through interleaving. This is because when $K$ is large enough, $\boldsymbol{h}_{i}, \boldsymbol{h}_{i+K}, \boldsymbol{h}_{i+2 K}, \cdots, \boldsymbol{h}_{i+(n-1) K}$ are roughly independent. Thus the codeword in codebook $\mathcal{C}_{i}$, which is transmitted over time instants $i, i+K, i+2 K, \cdots, i+(n-1) K$, essentially experiences a memoryless channel. This also suggests that as $K$ goes to infinity, the single-letter lower bound (5) provides a correct estimate of the rate supported by this interleaved decision-oriented training scheme. We can see that the channel memory manifests itself in the lower bound [5] only through $\mathbb{E}\left(\boldsymbol{h}_{i} \mid\left\{\boldsymbol{h}_{k}+\frac{1}{x_{\min }} \boldsymbol{z}_{k}\right\}_{k=-\infty}^{i-1}\right)$. Furthermore, in [5], we can write $\boldsymbol{h}_{i}$ as the sum of two independent random variables: the coherent fading component $\mathbb{E}\left(\boldsymbol{h}_{i} \mid\left\{\boldsymbol{h}_{k}+\frac{1}{x_{\min }} \boldsymbol{z}_{k}\right\}_{k=-\infty}^{i-1}\right)$ which is known the the receiver, and the non-coherent fading component $\boldsymbol{h}_{i}-\mathbb{E}\left(\boldsymbol{h}_{i} \mid\left\{\boldsymbol{h}_{k}+\frac{1}{x_{\min }} \boldsymbol{z}_{k}\right\}_{k=-\infty}^{i-1}\right)$ which is unknown. Isolating the effect of channel memory facilitates the channel code design: we only need to design channel codes for memoryless fading channels with different coherent and non-coherent components, instead of designing different codes for channels with different memory structures.

To derive a single-letter upper bound on $C(\mathrm{SNR})$, we follow the approach in [6]. The capacity $C(\mathrm{SNR})$ is given by

$$
C(\mathrm{SNR})=\lim _{n \rightarrow \infty} \sup _{P_{\boldsymbol{x}^{n}} \in \mathcal{P}_{n}(\mathrm{SNR})} \frac{1}{n} I\left(\boldsymbol{x}^{n} ; \boldsymbol{y}^{n}\right) .
$$


By the chain rule,

$$
I\left(\boldsymbol{x}^{n} ; \boldsymbol{y}^{n}\right)=\sum_{k=1}^{n} I\left(\boldsymbol{x}^{n} ; \boldsymbol{y}_{k} \mid \boldsymbol{y}^{k-1}\right)
$$

We can upper-bound $I\left(\boldsymbol{x}^{n} ; \boldsymbol{y}_{k} \mid \boldsymbol{y}^{k-1}\right)$ as

$$
\begin{aligned}
I\left(\boldsymbol{x}^{n} ; \boldsymbol{y}_{k} \mid \boldsymbol{y}^{k-1}\right) & =I\left(\boldsymbol{x}^{n}, \boldsymbol{y}^{k-1} ; \boldsymbol{y}_{k}\right)-I\left(\boldsymbol{y}_{k} ; \boldsymbol{y}^{k-1}\right) \\
& \leq I\left(\boldsymbol{x}^{n}, \boldsymbol{y}^{k-1} ; \boldsymbol{y}_{k}\right) \\
& =I\left(\boldsymbol{x}^{k}, \boldsymbol{y}^{k-1} ; \boldsymbol{y}_{k}\right) \\
& \leq I\left(\boldsymbol{x}_{k}, \boldsymbol{h}_{k-1}+\frac{1}{\sqrt{\mathrm{SNR}}} \boldsymbol{z}_{k-1}, \cdots, \boldsymbol{h}_{1}+\frac{1}{\sqrt{\mathrm{SNR}}} \boldsymbol{z}_{1} ; \boldsymbol{y}_{k}\right) \\
& \leq I\left(\boldsymbol{x}_{k},\left\{\boldsymbol{h}_{t}+\frac{1}{\sqrt{\mathrm{SNR}}} \boldsymbol{z}_{t}\right\}_{t=-\infty}^{k-1} ; \boldsymbol{y}_{k}\right) .
\end{aligned}
$$

Since $\left(\boldsymbol{x}_{k}, \mathbb{E}\left(\boldsymbol{h}_{k} \mid\left\{\boldsymbol{h}_{t}+\frac{1}{\sqrt{\mathrm{SNR}}} \boldsymbol{z}_{t}\right\}_{t=-\infty}^{k-1}\right)\right)$ is a sufficient statistic for estimating $\boldsymbol{y}_{k}$ from $\left(\boldsymbol{x}_{k},\left\{\boldsymbol{h}_{t}+\frac{1}{\sqrt{\mathrm{SNR}}} \boldsymbol{z}_{t}\right\}_{t=-\infty}^{k-1}\right)$, it follows that

$$
I\left(\boldsymbol{x}_{k},\left\{\boldsymbol{h}_{t}+\frac{1}{\sqrt{\mathrm{SNR}}} \boldsymbol{z}_{t}\right\}_{t=-\infty}^{k-1} ; \boldsymbol{y}_{k}\right)=I\left(\boldsymbol{x}_{k}, \mathbb{E}\left(\boldsymbol{h}_{k} \mid\left\{\boldsymbol{h}_{t}+\frac{1}{\sqrt{\mathrm{SNR}}} \boldsymbol{z}_{t}\right\}_{t=-\infty}^{k-1}\right) ; \boldsymbol{y}_{k}\right) .
$$

Note that by the block stationarity of the fading process,

$$
I\left(\boldsymbol{x}_{k}, \mathbb{E}\left(\boldsymbol{h}_{k} \mid\left\{\boldsymbol{h}_{t}+\frac{1}{\sqrt{\mathrm{SNR}}} \boldsymbol{z}_{t}\right\}_{t=-\infty}^{k-1}\right) ; \boldsymbol{y}_{k}\right)
$$

depends on $k$ only through $(k \bmod T)$. Therefore, we obtain the single-letter upper bound

$$
C(\mathrm{SNR}) \leq \frac{1}{T} \sum_{k=1}^{T} \sup _{P_{\boldsymbol{x}_{k}} \in \mathcal{P}_{1}(\mathrm{SNR})} I\left(\boldsymbol{x}_{k}, \mathbb{E}\left(\boldsymbol{h}_{k} \mid\left\{\boldsymbol{h}_{t}+\frac{1}{\sqrt{\mathrm{SNR}}} \boldsymbol{z}_{t}\right\}_{t=-\infty}^{k-1}\right) ; \boldsymbol{y}_{k}\right) .
$$

\section{B. Asymptotic Analysis}

Now we proceed to show that the lower bound (5) and upper bound (8) together characterize the asymptotic behavior of $C(\mathrm{SNR})$ in the high SNR regime.

Lemma 1: For every $\xi \in\left[\xi_{0}, \xi_{1}\right]$, let $A(\xi)$ be an $M \times M$ symmetric positive semidefinite matrix. We have

$$
\lim _{\epsilon \rightarrow 0} \frac{\int_{\xi_{0}}^{\xi_{1}} \log \operatorname{det}\left[A(\xi)+\epsilon I_{M}\right] \mathrm{d} \xi}{\log \epsilon}=\sum_{i=0}^{M}(M-i) \mu(\operatorname{rank}(A(\xi))=i) .
$$

where $\mu(\operatorname{rank}(A(\xi))=i)$ is the Lebesgue measure of the set $\{\xi: \operatorname{rank}(A(\xi))=i\}$.

For the special case where $A(\xi)=A$ for all $\xi \in\left[\xi_{0}, \xi_{1}\right]$ and $\xi_{1}-\xi_{0}=1$, we get

Proof: See Appendix [I]

$$
\lim _{\epsilon \rightarrow 0} \frac{\log \operatorname{det}\left[A+\epsilon I_{M}\right]}{\log \epsilon}=M-\operatorname{rank}(A) \text {. }
$$

Lemma 2 ( [6], Sec. V): If $\boldsymbol{x}$ is uniformly distributed over the set $\left\{z \in \mathbb{C}: \frac{\sqrt{\operatorname{SNR}}}{2} \leq|z| \leq \sqrt{\mathrm{SNR}}\right\}, \widehat{\boldsymbol{h}} \sim \mathcal{C N}\left(0, \mathbb{E}|\widehat{\boldsymbol{h}}|^{2}\right)$, $\widetilde{\boldsymbol{h}} \sim \mathcal{C N}\left(0, \mathbb{E}|\widetilde{\boldsymbol{h}}|^{2}\right), \boldsymbol{z} \sim \mathcal{C N}(0,1)$, and $\boldsymbol{x}, \widehat{\boldsymbol{h}}, \widetilde{\boldsymbol{h}}, \widehat{\boldsymbol{z}}$ are all independent, then

$$
I(\boldsymbol{x} ;(\widehat{\boldsymbol{h}}+\widetilde{\boldsymbol{h}}) \boldsymbol{x}+\boldsymbol{z} \mid \widehat{\boldsymbol{h}}) \geq-\log \left(\mathbb{E}|\widetilde{\boldsymbol{h}}|^{2}+\frac{8}{5 \mathrm{SNR}}\right)+\log \left(1-\mathbb{E}|\widetilde{\boldsymbol{h}}|^{2}\right)-\gamma-\log \frac{5 e}{6}
$$


where $\gamma$ is the Euler constant.

Theorem 1: For the block-stationary Gaussian fading channel model given in (1),

$$
\lim _{\mathrm{SNR} \rightarrow \infty} \frac{C(\mathrm{SNR})}{\log \mathrm{SNR}}=\lim _{\mathrm{SNR} \rightarrow \infty} \frac{-\log \operatorname{det}[\Sigma(\mathrm{SNR})]}{T \log \mathrm{SNR}}=\frac{1}{2 \pi T} \sum_{i=0}^{T}(T-i) \mu\left(\operatorname{rank}\left(S\left(e^{j \omega}\right)\right)=i\right) .
$$

Remark 3: The second equality in (10) follows from (2) and Lemma 1

Proof: Below we provide an intuitive explanation of this theorem based on the lower bound (5). The details of the proof are left to Appendix

In the lower bound (5), let $\boldsymbol{x}_{i}$ be uniformly distributed over the set $\left\{z \in \mathbb{C}: \frac{\sqrt{\mathrm{SNR}}}{2} \leq|z| \leq \sqrt{\mathrm{SNR}}\right\}$, and write $\boldsymbol{h}_{i}$ as $\boldsymbol{h}_{i}=\widehat{\boldsymbol{h}}_{i}+\widetilde{\boldsymbol{h}}_{i}$ where $\widehat{\boldsymbol{h}}_{i}=\mathbb{E}\left(\boldsymbol{h}_{i} \mid\left\{\boldsymbol{h}_{k}+\frac{2}{\sqrt{S N R}} \boldsymbol{z}_{k}\right\}_{k=-\infty}^{i-1}\right)$. Suppose $\mathbb{E}\left|\widetilde{\boldsymbol{h}}_{i}\right|^{2} \approx \mathrm{SNR}^{-r_{i}}, i=1,2, \cdots, T$. We can then write $\boldsymbol{y}_{i}$ as $\boldsymbol{y}_{i}=\widehat{\boldsymbol{h}}_{i} \boldsymbol{x}_{i}+\boldsymbol{w}_{i}$ where $\boldsymbol{w}_{i}=\widetilde{\boldsymbol{h}}_{i} \boldsymbol{x}_{i}+\boldsymbol{z}_{i}$ with $\mathbb{E}\left|\boldsymbol{w}_{i}\right|^{2} \approx \mathrm{SNR}^{1-r_{i}}$. By viewing $\boldsymbol{y}_{i}$ as the output of a coherent fading channel with the fading $\widehat{\boldsymbol{h}}_{i}$ known at the receiver and noise $\boldsymbol{w}_{i}$, we get

$$
\begin{aligned}
I\left(\boldsymbol{x}_{i} ; \boldsymbol{h}_{i} \boldsymbol{x}_{i}+\boldsymbol{z}_{i} \mid \mathbb{E}\left(\boldsymbol{h}_{i} \mid\left\{\boldsymbol{h}_{k}+\frac{2}{\sqrt{\mathrm{SNR}}} \boldsymbol{z}_{k}\right\}_{k=-\infty}^{i-1}\right)\right) & =I\left(\boldsymbol{x}_{i} ; \widehat{\boldsymbol{h}}_{i} \boldsymbol{x}_{i}+\boldsymbol{w}_{i} \mid \widehat{\boldsymbol{h}}_{i}\right) \\
& \approx \log \frac{\mathrm{SNR}}{\mathrm{SNR}^{1-r_{i}}} \\
& =r_{i} \log \mathrm{SNR} .
\end{aligned}
$$

Thus the lower bound (5) can be approximated by

$$
\frac{1}{T} \sum_{i=1}^{T} I\left(\boldsymbol{x}_{i} ; \boldsymbol{h}_{i} \boldsymbol{x}_{i}+\boldsymbol{z}_{i} \mid \mathbb{E}\left(\boldsymbol{h}_{i} \mid\left\{\boldsymbol{h}_{k}+\frac{2}{\sqrt{\mathrm{SNR}}} \boldsymbol{z}_{k}\right\}_{k=-\infty}^{i-1}\right)\right) \approx \frac{1}{T} \sum_{i=1}^{T} r_{i} \log \mathrm{SNR} .
$$

We then complete the proof by showing that $\sum_{i=1}^{T} r_{i}$ is related to the matrix spectral density function $S\left(e^{j \omega}\right)$ by the equation

$$
\sum_{i=1}^{T} r_{i}=\frac{1}{2 \pi} \sum_{i=0}^{T}(T-i) \mu\left(\operatorname{rank}\left(S\left(e^{j \omega}\right)\right)=i\right)
$$

Theorem 1 generalizes many previous results on the noncoherent capacity for Gaussian channels in the high SNR regime as we illustrate in the following subsection.

\section{Previous Results as Special Cases of Theorem $\square$}

\section{Example 1: Constant Fading within Block}

For the special case where the fading remains constant within a block, i.e., $\boldsymbol{h}_{k T+1}=\boldsymbol{h}_{k T+2}=\cdots=\boldsymbol{h}_{k T+T}$, for all $k \in \mathbb{Z}$, all the entries of $R(i)$ for any fixed $i$ are identical. This implies that, for any fixed $\omega$, all the entries of $S\left(e^{j \omega}\right)$ are identical, which we shall denote by $s\left(e^{j \omega}\right)$. It is easy to see that $s\left(e^{j \omega}\right)$ is essentially the spectral 
density function of $\left\{\boldsymbol{h}_{k T}\right\}_{k}$. The rank of $S\left(e^{j \omega}\right)$ is 1 if $s\left(e^{j \omega}\right)>0$, and is 0 if $s\left(e^{j \omega}\right)=0$. We therefore have

$$
\begin{aligned}
\lim _{\mathrm{SNR} \rightarrow \infty} \frac{C(\mathrm{SNR})}{\log \mathrm{SNR}} & =\frac{1}{2 \pi T} \sum_{i=0}^{T}(T-i) \mu\left(\operatorname{rank}\left(S\left(e^{j \omega}\right)\right)=i\right) \\
& =\frac{1}{2 \pi T} \sum_{i=0}^{1}(T-i) \mu\left(\operatorname{rank}\left(S\left(e^{j \omega}\right)\right)=i\right) \\
& =1-\frac{\mu\left(s\left(e^{j \omega}\right)>0\right)}{2 \pi T} .
\end{aligned}
$$

When $T=1$, we recover the result in [6] that

$$
\lim _{\mathrm{SNR} \rightarrow \infty} \frac{C(\mathrm{SNR})}{\log \mathrm{SNR}}=\frac{\mu\left(s\left(e^{j \omega}\right)=0\right)}{2 \pi}
$$

which illustrates the effect of large timescale correlation of the fading process on the pre-log term of the channel capacity in the high SNR regime. When the fading is independent from block to block, we have $\mu\left(s\left(e^{j \omega}\right)>0\right)=2 \pi$, and thus recover the result in [1], [2] that

$$
\lim _{\mathrm{SNR} \rightarrow \infty} \frac{C(\mathrm{SNR})}{\log \mathrm{SNR}}=\frac{T-1}{T}
$$

which illustrates the effect of short timescale correlation of the fading process on the pre-log term of the capacity at high SNR.

Example 2: Time-Selectivity within Block

In this example, we recover the main result in [7] concerning the case where rank deficiency is caused purely by the correlation within a block.

If $\operatorname{rank}(\Sigma(\infty))=\operatorname{rank}(R(0))$, then $^{1}$

$$
\lim _{\mathrm{SNR} \rightarrow \infty} \frac{C(\mathrm{SNR})}{\log \mathrm{SNR}}=\frac{T-\operatorname{rank}(R(0))}{T}
$$

To prove (12), we first note that

$$
\begin{aligned}
\Sigma(\infty)+\frac{1}{\mathrm{SNR}} I_{T} & =\operatorname{var}\left(\left(\boldsymbol{h}_{1}, \boldsymbol{h}_{2}, \cdots, \boldsymbol{h}_{T}\right)^{\top} \mid\left\{\boldsymbol{h}_{t}\right\}_{k=-\infty}^{0}\right)+\frac{1}{\mathrm{SNR}} I_{T} \\
& =\operatorname{var}\left(\left(\boldsymbol{h}_{1}+\frac{1}{\sqrt{\mathrm{SNR}}} \boldsymbol{z}_{1}, \cdots, \boldsymbol{h}_{T}+\frac{1}{\sqrt{\mathrm{SNR}}} \boldsymbol{z}_{T}\right)^{\top} \mid\left\{\boldsymbol{h}_{k}\right\}_{k=-\infty}^{0}\right) \\
& \preccurlyeq \operatorname{var}\left(\left(\boldsymbol{h}_{1}+\frac{1}{\sqrt{\mathrm{SNR}}} \boldsymbol{z}_{1}, \cdots, \boldsymbol{h}_{T}+\frac{1}{\sqrt{\mathrm{SNR}}} \boldsymbol{z}_{T}\right)^{\top} \mid\left\{\boldsymbol{h}_{k}+\frac{1}{\sqrt{\mathrm{SNR}}} \boldsymbol{z}_{k}\right\}_{k=-\infty}^{0}\right) \\
& =\Sigma(\mathrm{SNR}) \\
& \preccurlyeq \operatorname{var}\left(\left(\boldsymbol{h}_{1}+\frac{1}{\sqrt{\mathrm{SNR}}} \boldsymbol{z}_{1}, \cdots, \boldsymbol{h}_{T}+\frac{1}{\sqrt{\mathrm{SNR}}} \boldsymbol{z}_{T}\right)^{\top}\right) \\
& =R(0)+\frac{1}{\mathrm{SNR}} I_{T} .
\end{aligned}
$$

We therefore have the bound

$$
\lim _{\mathrm{SNR} \rightarrow \infty} \frac{\log \operatorname{det}\left[\Sigma(\infty)+\frac{1}{\mathrm{SNR}} I_{T}\right]}{\log \mathrm{SNR}} \leq \lim _{\mathrm{SNR} \rightarrow \infty} \frac{\log \operatorname{det} \Sigma(\mathrm{SNR})}{\log \mathrm{SNR}} \leq \lim _{\mathrm{SNR} \rightarrow \infty} \frac{\log \operatorname{det}\left[R(0)+\frac{1}{\mathrm{SNR}} I_{T}\right]}{\log \mathrm{SNR}}
$$

\footnotetext{
${ }^{1}$ The condition $\operatorname{rank}(\Sigma(\infty))=\operatorname{rank}(R(0))$ is satisfied, for instance, when the fading process is independent from block to block.
} 
By Lemma 11.

$$
\begin{aligned}
\lim _{\mathrm{SNR} \rightarrow \infty} \frac{\log \operatorname{det}\left[\Sigma(\infty)+\frac{1}{\mathrm{SNR}} I_{T}\right]}{\log \mathrm{SNR}} & =\lim _{\mathrm{SNR} \rightarrow \infty} \frac{\log \operatorname{det}\left[R(0)+\frac{1}{\mathrm{SNR}} I_{T}\right]}{\log \mathrm{SNR}} \\
& =-T+\operatorname{rank}(R(0)
\end{aligned}
$$

which implies that

$$
\lim _{\mathrm{SNR} \rightarrow \infty} \frac{\log \operatorname{det} \Sigma(\mathrm{SNR})}{\log \mathrm{SNR}}=-T+\operatorname{rank}(R(0) .
$$

Therefore, by Theorem 1

$$
\begin{aligned}
\lim _{\mathrm{SNR} \rightarrow \infty} \frac{C(\mathrm{SNR})}{\log \mathrm{SNR}} & =\lim _{\mathrm{SNR} \rightarrow \infty} \frac{-\log \operatorname{det} \Sigma(\mathrm{SNR})}{T \log \mathrm{SNR}} \\
& =\frac{T-\operatorname{rank}(R(0))}{T} .
\end{aligned}
$$

It is worth noting that in this case the pre-log term of the capacity can be achieved by a scheme simpler than the aforementioned interleaved decision-oriented training scheme. Suppose the rank of $R(0)$ is $Q$, so that $R(0)$ has $Q \times Q$ positive definite principal submatrix. Without loss of generality, suppose this submatrix is the covariance matrix of $\left(\boldsymbol{h}_{1}, \boldsymbol{h}_{2}, \cdots, \boldsymbol{h}_{Q}\right)^{T}$. Then $\boldsymbol{h}_{k T+i}$ can be represented as a linear combination of $\boldsymbol{h}_{k T+1}, \boldsymbol{h}_{k T_{2}}, \cdots, \boldsymbol{h}_{k T+Q}$ for any $k \in \mathbb{Z}$ and $i \in\{Q+1, Q+2, \cdots, T\}$. The simpler scheme is described as follows:

The transmitter sends deterministic training symbols with maximum power at time instants $k T+1, k T+$ $2, \cdots, k T+Q$, i.e., $\boldsymbol{x}_{k T+1}=\boldsymbol{x}_{k T+2}=\cdots=\boldsymbol{x}_{k T+Q}=\sqrt{\mathrm{SNR}}$, where $k=0,1,2, \cdots$. The receiver can form the MMSE estimates $\mathbb{E}\left(\boldsymbol{h}_{k T+i} \mid\left\{\boldsymbol{h}_{k T+j}+\frac{1}{\sqrt{\mathrm{SNR}}} \boldsymbol{z}_{k T+j}\right\}_{j=1}^{Q}\right)$, for $i=Q+1, Q+2, \cdots, T$ and $k=0,1,2, \cdots$. Clearly, we have

$$
\operatorname{var}\left(\boldsymbol{h}_{k T+i} \mid\left\{\boldsymbol{h}_{k T+j}+\frac{1}{\sqrt{\mathrm{SNR}}} \boldsymbol{z}_{k T+j}\right\}_{j=1}^{Q}\right)=O\left(\frac{1}{\mathrm{SNR}}\right) .
$$

With the side information $\left\{\mathbb{E}\left(\boldsymbol{h}_{k T+i} \mid\left\{\boldsymbol{h}_{k T+j}+\frac{1}{\sqrt{\mathrm{SNR}}} \boldsymbol{z}_{k T+j}\right\}_{j=1}^{Q}\right)\right\}_{k=0}^{\infty}$ at the receiver, we can communicate reliably at time instants $i, T+i, 2 T+i, \cdots$ with rate at least $I\left(\boldsymbol{x}_{i} ; \boldsymbol{y}_{i} \mid \mathbb{E}\left(\boldsymbol{h}_{i} \mid\left\{\boldsymbol{h}_{j}+\frac{1}{\sqrt{\mathrm{SNR}}} \boldsymbol{z}_{j}\right\}_{j=1}^{Q}\right)\right)$. Let $\boldsymbol{x}_{i}$ be uniformly distributed over the set $\{z \in \mathbb{C}: \mathrm{SNR} / 2 \leq\|z\| \leq \mathrm{SNR}\}$. By Lemma 2

$$
\begin{aligned}
I\left(\boldsymbol{x}_{i} ; \boldsymbol{y}_{i} \mid \mathbb{E}\left(\boldsymbol{h}_{i} \mid\left\{\boldsymbol{h}_{j}+\frac{1}{\sqrt{\mathrm{SNR}}} \boldsymbol{z}_{j}\right\}_{j=1}^{Q}\right)\right) \geq & -\log \left[\operatorname{var}\left(\boldsymbol{h}_{i} \mid\left\{\boldsymbol{h}_{j}+\frac{1}{\sqrt{\mathrm{SNR}}} \boldsymbol{z}_{j}\right\}_{j=1}^{Q}\right)+\frac{8}{5 \mathrm{SNR}}\right] \\
& +\log \left(1-\operatorname{var}\left(\boldsymbol{h}_{i} \mid\left\{\boldsymbol{h}_{j}+\frac{1}{\sqrt{\mathrm{SNR}}} \boldsymbol{z}_{j}\right\}_{j=1}^{Q}\right)\right)-\gamma-\log \frac{5 e}{6} \\
= & \log \mathrm{SNR}+o(\log \mathrm{SNR}) .
\end{aligned}
$$

Threfore, the overall rate is lower-bounded by

$$
\begin{aligned}
\frac{1}{T} \sum_{i=Q+1}^{T} I\left(\boldsymbol{x}_{i} ; \boldsymbol{y}_{i} \mid \mathbb{E}\left(\boldsymbol{h}_{i} \mid\left\{\boldsymbol{h}_{j}+\frac{1}{\sqrt{\mathrm{SNR}}} \boldsymbol{z}_{j}\right\}_{j=1}^{Q}\right)\right) & =\frac{T-Q}{T} \log \mathrm{SNR}+o(\log \mathrm{SNR}) \\
& =\frac{T-\operatorname{rank}(R(0))}{T} \log \mathrm{SNR}+o(\log \mathrm{SNR})
\end{aligned}
$$


and the pre-log term is achieved. This scheme has the following obvious advantages over the interleaved decisionoriented training scheme: (i) channel estimation and communication are completely decoupled; and (ii) channel estimation is done locally since the estimate $\mathbb{E}\left(\boldsymbol{h}_{k T+i} \mid\left\{\boldsymbol{h}_{k T+j}+\frac{1}{\sqrt{\mathrm{SNR}}} \boldsymbol{z}_{k T+j}\right\}_{j=1}^{Q}\right), i=Q+1, Q+2, \cdots, T$, only depends $\boldsymbol{h}_{k T+1}, \boldsymbol{h}_{k T+2}, \cdots, \boldsymbol{h}_{k T+Q}$.

\section{Regular Block-Stationary Process}

The following theorem generalizes [3, Corollary 4.42] for regular Gaussian fading processes to the block-stationary case.

Theorem 2: If $\operatorname{det}(\Sigma(\infty))>0$, then

$\lim _{\mathrm{SNR} \rightarrow \infty}[C(\mathrm{SNR})-\log \log \mathrm{SNR}]=-1-\gamma-\frac{1}{T} \log \operatorname{det}(\Sigma(\infty))=-1-\gamma-\frac{1}{2 \pi T} \int_{-\pi}^{\pi} \log \operatorname{det}\left[S\left(e^{j \omega}\right)\right] \mathrm{d} \omega$.

Remark 4: The second equality in (13) follows from (3).

Proof: See Appendix IV

Example 3: Gauss-Markov Process

Suppose $\left\{\boldsymbol{h}_{t}\right\}_{t=-\infty}^{\infty}$ is a Gauss-Markov process with $E\left(\boldsymbol{h}_{t+1} \boldsymbol{h}_{t}^{*}\right)=\rho_{1}$ if $(t \bmod T)=0$, and $=\rho_{2}$ otherwise. Here $\rho_{1}, \rho_{2}$ are complex numbers with $\max \left(\left|\rho_{1}\right|,\left|\rho_{2}\right|\right)<1$. In this case, we have

$$
\operatorname{det}(\Sigma(\infty))=\left(1-\left|\rho_{1}\right|^{2}\right)\left(1-\left|\rho_{2}\right|^{2}\right)^{T-1} .
$$

Therefore, by Theorem 2

$$
\lim _{\mathrm{SNR} \rightarrow \infty}[C(\mathrm{SNR})-\log \log \mathrm{SNR}]=-1-\gamma-\log \left(1-\left|\rho_{2}\right|^{2}\right)-\frac{\log \left(1-\left|\rho_{1}\right|^{2}\right)-\log \left(1-\left|\rho_{1}\right|^{2}\right)}{T} .
$$

\section{Symbol-By-Symbol Stationary FAding Model}

For simplicity, we assume in this section that the fading process is symbol-by-symbol stationary, i.e., $T=1$. In this case, Theorem 1 is specialized to Equation (11).

\section{A. Best- and Worst-Case Spectral Densities}

We can see that two fading processes with spectral density functions $s_{1}\left(e^{j \omega}\right)$ and $s_{2}\left(e^{j \omega}\right)$ can induce the same pre-log term in the high SNR regime as long as $\mu\left(s_{1}\left(e^{j \omega}\right)=0\right)=\mu\left(s_{2}\left(e^{j \omega}\right)=0\right)$. But in the non-asymptotic regime, the capacities of these two channels may behave very differently. So, for a fixed $\mu\left(s\left(e^{j \omega}\right)=0\right)$, it is natural to ask the question: which spectral density function $s\left(e^{j \omega}\right)$ gives the largest (or smallest) channel capacity at a given SNR? This question is difficult to answer since we do not have a closed-form expression for noncoherent channel capacity. We therefore turn to the lower bound (5) to formulate a closely-related problem.

When $T=1$, the lower bound (5) can be reduced to

$$
C(\mathrm{SNR}) \geq I\left(\boldsymbol{x}_{1} ; \boldsymbol{h}_{1} \boldsymbol{x}_{1}+\boldsymbol{z}_{1} \mid \mathbb{E}\left(\boldsymbol{h}_{1} \mid\left\{\boldsymbol{h}_{k}+\frac{1}{x_{\min }} \boldsymbol{z}_{1}\right\}_{k=-\infty}^{0}\right)\right) .
$$


We can see that the lower bound (14) depends on $s\left(e^{j \omega}\right)$ only through $\mathbb{E}\left(\boldsymbol{h}_{1} \mid\left\{\boldsymbol{h}_{k}+\frac{1}{x_{\min }} \boldsymbol{z}_{1}\right\}_{k=-\infty}^{0}\right)$. Furthermore, if we fix the input distribution $p\left(\boldsymbol{x}_{1}\right)$, then

$$
\left.\operatorname{var}\left(\boldsymbol{h}_{1} \mid\left\{\boldsymbol{h}_{k}+\frac{1}{x_{\min }} \boldsymbol{z}_{1}\right\}_{k=-\infty}^{0}\right)\right|_{s_{1}\left(e^{j \omega}\right)} \leq\left.\operatorname{var}\left(\boldsymbol{h}_{1} \mid\left\{\boldsymbol{h}_{k}+\frac{1}{x_{\min }} \boldsymbol{z}_{1}\right\}_{k=-\infty}^{0}\right)\right|_{s_{2}\left(e^{j \omega}\right)}
$$

implies that

$$
\begin{aligned}
& \left.I\left(\boldsymbol{x}_{1} ; \boldsymbol{h}_{1} \boldsymbol{x}_{1}+\boldsymbol{z}_{1} \mid \mathbb{E}\left(\boldsymbol{h}_{1} \mid\left\{\boldsymbol{h}_{k}+\frac{1}{x_{\min }} \boldsymbol{z}_{1}\right\}_{k=-\infty}^{0}\right)\right)\right|_{s_{1}\left(e^{j \omega}\right)} \\
\geq & \left.I\left(\boldsymbol{x}_{1} ; \boldsymbol{h}_{1} \boldsymbol{x}_{1}+\boldsymbol{z}_{1} \mid \mathbb{E}\left(\boldsymbol{h}_{1} \mid\left\{\boldsymbol{h}_{k}+\frac{1}{x_{\min }} \boldsymbol{z}_{1}\right\}_{k=-\infty}^{0}\right)\right)\right|_{s_{2}\left(e^{j \omega}\right)} .
\end{aligned}
$$

We can therefore ask which $s\left(e^{j \omega}\right)$ gives the largest (or smallest) var $\left.\left(\boldsymbol{h}_{1} \mid\left\{\boldsymbol{h}_{k}+\frac{1}{x_{\min }} \boldsymbol{z}_{1}\right\}_{k=-\infty}^{0}\right)\right|_{s\left(e^{j \omega}\right)}$. More precisely, since

$$
\begin{aligned}
\left.\operatorname{var}\left(\boldsymbol{h}_{1} \mid\left\{\boldsymbol{h}_{k}+\frac{1}{x_{\min }} \boldsymbol{z}_{1}\right\}_{k=-\infty}^{0}\right)\right|_{s\left(e^{j \omega}\right)} & =\left.\operatorname{var}\left(\boldsymbol{h}_{1}+\frac{1}{x_{\min }} \boldsymbol{z}_{1} \mid\left\{\boldsymbol{h}_{k}+\frac{1}{x_{\min }} \boldsymbol{z}_{1}\right\}_{k=-\infty}^{0}\right)\right|_{s\left(e^{j \omega}\right)}-\frac{1}{x_{\min }^{2}} \\
& =\exp \left\{\frac{1}{2 \pi} \int_{-\pi}^{\pi} \log \left[s\left(e^{j \omega}\right)+\frac{1}{x_{\min }^{2}}\right] \mathrm{d} \omega\right\}-\frac{1}{x_{\min }^{2}},
\end{aligned}
$$

we can formulate the problem in the following form:

$$
\arg \max _{s\left(e^{j \omega}\right)}\left(\operatorname{or} \arg \min _{s\left(e^{j \omega}\right)}\right) \int_{-\pi}^{\pi} \log \left[s\left(e^{j \omega}\right)+\frac{1}{x_{\min }^{2}}\right] \mathrm{d} \omega
$$

subject to

$$
s\left(e^{j \omega}\right) \geq 0, \quad \frac{1}{2 \pi} \int_{-\pi}^{\pi} s\left(e^{j \omega}\right) \mathrm{d} \omega=1, \quad \mu\left(s\left(e^{j \omega}\right)=0\right)=\alpha .
$$

where $\alpha \in[0,2 \pi)$. Due to the strict concavity of $\log (\cdot)$, it is easy to show that the maximizers of the optimization problem (15) are the set of spectral density functions $s\left(e^{j \omega}\right)$ with the property:

$$
\mu\left(s\left(e^{j \omega}\right)=\frac{1}{2 \pi-\alpha}\right)=2 \pi-\alpha, \quad \mu\left(s\left(e^{j \omega}\right)=0\right)=\alpha .
$$

This solution has the following interpretation. Without constraints on the spectral density function, the worst fading process is the i.i.d. Gaussian process whose spectral density function is flat. With the constraint $\mu\left(s\left(e^{j \omega}\right)=0\right)=\alpha$, the spectral density function $s\left(e^{j \omega}\right)$ cannot be completely flat, but the worst fading process should have a spectral density function that is as flat as possible, i.e., the correlation in the time domain should be the weakest possible. Note that the solution does not depend on $x_{\min }$. We can use this fact to derive a universal lower bound on $C(\mathrm{SNR})$ for the class of spectral density functions $\left\{s\left(e^{j \omega}\right): \mu\left(s\left(e^{j \omega}\right)=0\right)=\alpha\right\}$, which has further implications for the high SNR asymptotic behavior of $C(\mathrm{SNR})$. Let $s_{\max }\left(e^{j \omega}\right)$ be a maximizer of (15). We have

$$
\left.\operatorname{var}\left(\boldsymbol{h}_{1} \mid\left\{\boldsymbol{h}_{k}+\frac{1}{x_{\min }} \boldsymbol{z}_{1}\right\}_{k=-\infty}^{0}\right)\right|_{s_{\max }\left(e^{j \omega}\right)}=\left(\frac{1}{2 \pi-\alpha}+\frac{1}{x_{\min }^{2}}\right)^{\frac{2 \pi-\alpha}{2 \pi}}\left(\frac{1}{x_{\min }^{2}}\right)^{\frac{\alpha}{2 \pi}}-\frac{1}{x_{\min }^{2}} \triangleq \phi\left(\alpha, x_{\min }\right) .
$$


For any spectral density function $s\left(e^{j \omega}\right)$ with $\mu\left(s\left(e^{j \omega}\right)=0\right)=\alpha$, we have

$$
\begin{aligned}
\left.C(\mathrm{SNR})\right|_{s\left(e^{j \omega}\right)} & \geq\left. I\left(\boldsymbol{x}_{1} ; \boldsymbol{h}_{1} \boldsymbol{x}_{1}+\boldsymbol{z}_{1} \mid \mathbb{E}\left(\boldsymbol{h}_{1} \mid\left\{\boldsymbol{h}_{k}+\frac{1}{x_{\min }} \boldsymbol{z}_{1}\right\}_{k=-\infty}^{0}\right)\right)\right|_{s_{\max }\left(e^{j \omega}\right)} \\
& =I(\boldsymbol{x} ;(\widehat{\boldsymbol{h}}+\widetilde{\boldsymbol{h}}) \boldsymbol{x}+\boldsymbol{z} \mid \widehat{\boldsymbol{h}})
\end{aligned}
$$

where $\boldsymbol{x}$ is uniformly distributed over the set $\left\{z \in \mathbb{C}: x_{\min } \leq|z| \leq \sqrt{\mathrm{SNR}}\right\}, \widehat{\boldsymbol{h}} \sim \mathcal{C N}\left(0,1-\phi\left(\alpha, x_{\min }\right)\right), \widetilde{\boldsymbol{h}} \sim$ $\mathcal{C N}\left(0, \phi\left(\alpha, x_{\min }\right)\right), \boldsymbol{z} \sim \mathcal{C N}(0,1)$, and $\boldsymbol{x}, \widehat{\boldsymbol{h}}, \widetilde{\boldsymbol{h}}, \widehat{\boldsymbol{z}}$ are all independent. We can further optimize over $p(\boldsymbol{x})$ to tighten the lower bound (16).

The minimizers of (15) do not exist. Consider the following set spectral density functions $\left\{s_{\theta}\left(e^{j \omega}\right)\right\}_{\theta}$ given by

$$
s_{\theta}\left(e^{j \omega}\right)= \begin{cases}0 & |\omega| \leq \frac{\alpha}{2} \\ \frac{1}{\theta} & |\omega| \in\left(\frac{\alpha}{2}, \pi-\frac{1}{2 \theta}\right] \\ \frac{2 \pi \theta^{2}-2 \pi \theta+\alpha \theta+1}{\theta} & |\omega| \in\left(\pi-\frac{1}{2 \theta}, \pi\right]\end{cases}
$$

where $\theta \geq \theta_{0}$ with

$$
\theta_{0}= \begin{cases}\frac{1}{2 \pi-\alpha} & (2 \pi-\alpha)^{2}<8 \pi \\ \frac{2 \pi-\alpha+\sqrt{(2 \pi-\alpha)^{2}-8 \pi}}{4 \pi} & (2 \pi-\alpha)^{2} \geq 8 \pi\end{cases}
$$

We can compute

$$
\begin{aligned}
& \lim _{\theta \rightarrow \infty} \int_{-\pi}^{\pi} \log \left[s_{\theta}\left(e^{j \omega}\right)+\frac{1}{x_{\min }^{2}}\right] \mathrm{d} \omega \\
= & \lim _{\theta \rightarrow \infty}\left[-2 \alpha \log x_{\min }+\left(2 \pi-\alpha-\frac{1}{\theta}\right) \log \left(\frac{1}{\theta}+\frac{1}{x_{\min }^{2}}\right)+\frac{1}{\theta} \log \left(\frac{2 \pi \theta^{2}-2 \pi \theta+\alpha \theta+1}{\theta}+x_{\min }^{2}\right)\right] \\
= & -4 \pi \log x_{\min } .
\end{aligned}
$$

Note that $\int_{-\pi}^{\pi} \log \left[s\left(e^{j \omega}\right)+\frac{1}{x_{\min }^{2}}\right] \mathrm{d} \omega<-4 \pi \log x_{\min }$. Therefore, as $\theta$ goes to infinity, $\int_{-\pi}^{\pi} \log \left[s\left(e^{j \omega}\right)+\frac{1}{x_{\min }^{2}}\right] \mathrm{d} \omega$ approaches the lower bound that is not attainable by any spectral density function. Intuitively, the fading process associated with $s_{\theta}\left(e^{j \omega}\right)$ becomes more and more deterministic as $\theta$ gets larger, and it can be verified that

$$
\left.\lim _{\theta \rightarrow \infty} \operatorname{var}\left(\boldsymbol{h}_{1} \mid\left\{\boldsymbol{h}_{k}+\frac{1}{x_{\min }} \boldsymbol{z}_{k}\right\}_{k=-\infty}^{0}\right)\right|_{s_{\theta}\left(e^{j \omega}\right)}=0 .
$$

This result has interesting implications for the channel capacity.

Proposition 1: For any $r \geq 0$,

$$
\liminf _{\mathrm{SNR} \rightarrow \infty, \theta=\mathrm{SNR}^{r}} \frac{\left.C(\mathrm{SNR})\right|_{s_{\theta}\left(e^{j \omega}\right)}}{\log \mathrm{SNR}} \geq \frac{\alpha+\min (r, 1)(2 \pi-\alpha)}{2 \pi} .
$$

If $r \geq 1$, then

Proof: See Appendix V

$$
\lim _{\mathrm{SNR} \rightarrow \infty, \theta=\mathrm{SNR}^{r}} \frac{\left.C(\mathrm{SNR})\right|_{s_{\theta}\left(e^{j \omega}\right)}}{\log \mathrm{SNR}}=1
$$


Remark 5: Although by Theorem 1 for any fixed $\theta$, the ratio between $\left.C(\mathrm{SNR})\right|_{s_{\theta}\left(e^{j \omega}\right)}$ and log SNR converges to $\frac{\alpha}{2 \pi}$, Proposition 1 says that the convergence is not uniform with respect to $\theta$. This is intuitively clear because when $\theta$ is large, we have $s_{\theta}\left(e^{j \omega}\right) \approx 0$ for $|\omega| \in\left[0, \pi-\frac{1}{2 \theta}\right]$. Therefore it can be expected that for a large range of SNR, the channel capacity $\left.C(\mathrm{SNR})\right|_{s_{\theta}\left(e^{j \omega}\right)}$ behaves like $\left(1-\frac{1}{\pi \theta}\right) \log \mathrm{SNR}$, which could be significantly larger than $\frac{\alpha}{2 \pi} \log$ SNR. For the extreme case where $\alpha=0$, by Theorem 2 for any fixed $\theta$, the capacity $\left.C(\operatorname{SNR})\right|_{s\left(e^{j \omega}\right)}$ grows like $\log \log$ SNR at high SNR. But Proposition 1 implies that even in this extreme case, the capacity $\left.C(\operatorname{SNR})\right|_{s_{\theta}\left(e^{j \omega}\right)}$ of some $\theta$ can grow linearly with $\log$ SNR for a large range of SNR. This is consistent with the result in [10] where is was shown that for the Gauss-Markov process with $E\left(\boldsymbol{h}_{i+1} \boldsymbol{h}_{i}^{*}\right)=\rho$, the capacity $C(\mathrm{SNR})$ grows like log SNR for a wide range of SNR levels if $|\rho|$ is close 1 . An intuitive explanation is that if $|\rho|$ is close 1 , the spectrum

$$
s\left(e^{j \omega}\right)=\frac{1-|\rho|^{2}}{1-2 \operatorname{Re}\left(\rho e^{-j \omega}\right)+|\rho|^{2}}
$$

is approximately zero for all $\omega$ except those around zero, and we can expect from equation (11) that $C(\mathrm{SNR})$ should grow like $\log$ SNR for a wide range of SNR. But it should be noted that as opposed to a Gauss-Markov process, a general Gaussian stationary process cannot be characterized by a single parameter, and the behavior of $C($ SNR) can be much more complicated as shown in the following example.

Example 4: Consider the spectral density function

$$
s_{\theta}\left(e^{j \omega}\right)= \begin{cases}\epsilon_{1} & |\omega| \leq \pi \alpha_{1} \\ \epsilon_{2} & |\omega| \in\left(\pi \alpha_{1}, \pi \alpha_{2}\right] \\ \frac{1-\alpha_{1} \epsilon_{1}-\left(\alpha_{2}-\alpha_{1}\right) \epsilon_{2}}{1-\alpha_{2}} & |\omega| \in\left(\pi \alpha_{2}, \pi\right]\end{cases}
$$

where $0<\alpha_{1}<\alpha_{2}<1$, and $\epsilon_{1} \ll \epsilon_{2} \ll 1$ (Note: For two positive numbers $a$ and $b, a \ll b$ means $\frac{b}{a}$ is much greater than 1). We show in Appendix VI that $\frac{C(\mathrm{SNR})}{\log \mathrm{SNR}}$ is approximately equal to $\alpha_{2}$ for SNR $\leq \frac{1}{\epsilon_{2}}$, and gradually decreases to $\alpha_{1}$ as SNR approaches $\frac{1}{\epsilon_{1}}$.

This example shows that $\frac{C(\mathrm{SNR})}{\log \mathrm{SNR}}$ can be highly SNR dependent. For a regular Gaussian fading process, it may require unreasonably high (impractical) values of SNR in order for the noncoherent channel capacity $C$ (SNR) to grow like $\log \log \mathrm{SNR}$, and the behavior of $C(\mathrm{SNR})$ at moderate SNR levels may depend highly on the spectral density function.

Overall, the above analysis suggests that great caution should be exercised when using the asymptotic results in Theorem 1 and Theorem 2 to approximate the channel capacity $C(\mathrm{SNR})$ at a finite SNR level.

\section{B. Finite Codeword-length Behavior}

Although the asymptotic capacity results might yield over-pessimistic approximations such as a $\log \log$ SNR growth with SNR, they could also lead to over-optimistic conclusions. In the capacity analysis, it is assumed that the codeword is of infinite length. But when the length of codewords is finite, the situation can be dramatically different. By Fano's inequality, the communication rate $R$ is upper-bounded by

$$
R \leq \frac{I\left(\boldsymbol{x}^{n} ; \boldsymbol{y}^{n}\right)+1}{n\left(1-P_{e}\right)}
$$


where $n$ is the codeword length and $P_{e}$ is the decoding error probability. Suppose we fix $n$ and $P_{e}$, and let SNR go to infinity. For a symbol-by-symbol stationary Gaussian fading process, even if $\mu\left(s\left(e^{j \omega}\right)=0\right)>0$, the correlation matrix of the fading process over any finite block length can still be full-rank. Note that $\frac{1}{n} I\left(\boldsymbol{x}^{n} ; \boldsymbol{y}^{n}\right)$ is upperbounded by the capacity of a block-independent Gaussian fading channel with the correlation matrix of each block given by $\mathbb{E}\left[\boldsymbol{h}^{n}\left(\boldsymbol{h}^{n}\right)^{\dagger}\right]$. Since $\mathbb{E}\left[\boldsymbol{h}^{n}\left(\boldsymbol{h}^{n}\right)^{\dagger}\right]$ is full rank, it follows from Theorem 2 that $\frac{1}{n} I\left(\boldsymbol{x}^{n} ; \boldsymbol{y}^{n}\right)$, and hence $R$, grows at most like $\log \log$ SNR as SNR goes to infinity. Therefore, there is no nontrivial tradeoff between diversity and multiplexing in the sense of [11]. If we want $R$ to grow linearly with $\log$ SNR while having the decoding error probability $P_{e}$ bounded away from 1 , the codeword length $n$ must scale with SNR. It is of interest to determine how fast the codeword $n$ should scale with SNR in order to guarantee that the rate $R$ can grow as $\log$ SNR with the decoding error probability not approaching 1 . More precisely, letting the rate $R(\mathrm{SNR})$, codeword length $n(\mathrm{SNR})$ and decoding error probability $P_{e}(\mathrm{SNR})$ all depend on SNR, we wish to determine conditions on $n(\mathrm{SNR})$ to guarantee the existence of a sequence of codebooks (indexed by SNR) with rate $R(\mathrm{SNR})$ and codeword length $n(\mathrm{SNR})$ such that

$$
\liminf _{\mathrm{SNR} \rightarrow \infty} \frac{R(\mathrm{SNR})}{\log \mathrm{SNR}} \geq r
$$

and

$$
\limsup _{\mathrm{SNR} \rightarrow \infty} P_{e}(\mathrm{SNR}) \leq P_{e}
$$

where $r>0$ and $P_{e} \in(0,1)$.

Now we proceed to derive a necessary condition on the growth rate of $n(\mathrm{SNR})$. It follows by chain rule that

$$
I\left(\boldsymbol{x}^{n(\mathrm{SNR})} ; \boldsymbol{y}^{n(\mathrm{SNR})}\right)=\sum_{k=1}^{n(\mathrm{SNR})} I\left(\boldsymbol{x}^{n(\mathrm{SNR})} ; \boldsymbol{y}_{k} \mid \boldsymbol{y}^{k-1}\right)
$$

By [6, we can upper-bound $I\left(\boldsymbol{x}^{n(\mathrm{SNR})} ; \boldsymbol{y}_{k} \mid \boldsymbol{y}^{k-1}\right)$ as

$$
\begin{aligned}
& I\left(\boldsymbol{x}^{n(\mathrm{SNR})} ; \boldsymbol{y}_{k} \mid \boldsymbol{y}^{k-1}\right) \\
\leq & \sup _{P_{\boldsymbol{x}_{k}} \in \mathcal{P}_{1}(\mathrm{SNR})} I\left(\boldsymbol{x}_{k}, \boldsymbol{h}_{k-1}+\frac{1}{\sqrt{\mathrm{SNR}}} \boldsymbol{z}_{k-1}, \cdots, \boldsymbol{h}_{1}+\frac{1}{\sqrt{\mathrm{SNR}}} \boldsymbol{z}_{1} ; \boldsymbol{y}_{k}\right) \\
\leq & \sup _{P_{\boldsymbol{x}_{k}} \in \mathcal{P}_{1}(\mathrm{SNR})} I\left(\boldsymbol{x}_{k} ; \boldsymbol{y}_{k}\right)+\sup _{P_{\boldsymbol{x}_{k}} \in \mathcal{P}_{1}(\mathrm{SNR})} I\left(\boldsymbol{h}_{k-1}+\frac{1}{\sqrt{\mathrm{SNR}}} \boldsymbol{z}_{k-1}, \cdots, \boldsymbol{h}_{1}+\frac{1}{\sqrt{\mathrm{SNR}}} \boldsymbol{z}_{1} ; \boldsymbol{y}_{k} \mid \boldsymbol{x}_{k}\right)
\end{aligned}
$$

Since $\sup _{P_{\boldsymbol{x}_{k}} \in \mathcal{P}_{1}(\mathrm{SNR})} I\left(\boldsymbol{x}_{k} ; \boldsymbol{y}_{k}\right)=o(\log \mathrm{SNR})$, and

$$
\begin{aligned}
& I\left(\boldsymbol{h}_{k-1}+\frac{1}{\sqrt{\mathrm{SNR}}} \boldsymbol{z}_{k-1}, \cdots, \boldsymbol{h}_{1}+\frac{1}{\sqrt{\mathrm{SNR}}} \boldsymbol{z}_{1} ; \boldsymbol{y}_{k} \mid \boldsymbol{x}_{k}\right) \\
= & \mathbb{E}\left\{\log \left[\frac{1+\left|\boldsymbol{x}_{k}\right|^{2}}{1+\left|\boldsymbol{x}_{k}\right|^{2} \cdot \operatorname{var}\left(\boldsymbol{h}_{k} \mid\left\{\boldsymbol{h}_{v}+\frac{1}{\sqrt{\mathrm{SNR}}} \boldsymbol{z}_{v}\right\}_{v=1}^{k-1}\right)}\right]\right\} \\
\leq & \log \left[\frac{1+\mathrm{SNR}}{1+\mathrm{SNR} \cdot \operatorname{var}\left(\boldsymbol{h}_{k} \mid\left\{\boldsymbol{h}_{v}+\frac{1}{\sqrt{\mathrm{SNR}}} \boldsymbol{z}_{v}\right\}_{v=1}^{k-1}\right)}\right]
\end{aligned}
$$




$$
\begin{aligned}
& =\log \left(\frac{1+\mathrm{SNR}}{\mathrm{SNR}}\right)-\log \operatorname{var}\left(\boldsymbol{h}_{k}+\frac{1}{\sqrt{\mathrm{SNR}}} \boldsymbol{z}_{k} \mid\left\{\boldsymbol{h}_{v}+\frac{1}{\sqrt{\mathrm{SNR}}} \boldsymbol{z}_{v}\right\}_{v=1}^{k-1}\right) \\
& \leq \log \left(\frac{1+\mathrm{SNR}}{\mathrm{SNR}}\right)-\log \operatorname{var}\left(\boldsymbol{h}_{0}+\frac{1}{\sqrt{\mathrm{SNR}}} \boldsymbol{z}_{0} \mid\left\{\boldsymbol{h}_{v}+\frac{1}{\sqrt{\mathrm{SNR}}} \boldsymbol{z}_{v}\right\}_{v=-n(\mathrm{SNR})}^{-1}\right),
\end{aligned}
$$

it follows by Fano's inequality and the condition $\lim _{\sup } \operatorname{SNR} \rightarrow \infty_{e} P_{e}(\mathrm{SNR}) \leq P_{e}$ that

$$
\begin{aligned}
\liminf _{\mathrm{SNR} \rightarrow \infty} \frac{R(\mathrm{SNR})}{\log \mathrm{SNR}} & \leq \liminf _{\mathrm{SNR} \rightarrow \infty} \frac{I\left(\boldsymbol{x}^{n(\mathrm{SNR})} ; \boldsymbol{y}^{n(\mathrm{SNR})}\right)+1}{n(\mathrm{SNR})\left(1-P_{e}(\mathrm{SNR})\right) \log \mathrm{SNR}} \\
& \leq \liminf _{\mathrm{SNR} \rightarrow \infty} \frac{-\log \operatorname{var}\left(\boldsymbol{h}_{0}+\frac{1}{\sqrt{\mathrm{SNR}}} \boldsymbol{z}_{0} \mid\left\{\boldsymbol{h}_{v}+\frac{1}{\sqrt{\mathrm{SNR}}} \boldsymbol{z}_{v}\right\}_{v=-n(\mathrm{SNR})}^{-1}\right)}{\left(1-P_{e}(\mathrm{SNR})\right) \log \mathrm{SNR}} \\
& \leq \liminf _{\mathrm{SNR} \rightarrow \infty} \frac{-\log \operatorname{var}\left(\boldsymbol{h}_{0}+\frac{1}{\sqrt{\mathrm{SNR}}} \boldsymbol{z}_{0} \mid\left\{\boldsymbol{h}_{v}+\frac{1}{\sqrt{\mathrm{SNR}}} \boldsymbol{z}_{v}\right\}_{v=-n(\mathrm{SNR})}^{-1}\right)}{\left(1-P_{e}\right) \log \mathrm{SNR}} .
\end{aligned}
$$

Therefore, in order for

$$
\liminf _{\mathrm{SNR} \rightarrow \infty} \frac{R(\mathrm{SNR})}{\log \mathrm{SNR}} \geq r
$$

we must have

$$
\liminf _{\mathrm{SNR} \rightarrow \infty} \frac{-\log \operatorname{var}\left(\boldsymbol{h}_{0}+\frac{1}{\sqrt{\mathrm{SNR}}} \boldsymbol{z}_{0} \mid\left\{\boldsymbol{h}_{v}+\frac{1}{\sqrt{\mathrm{SNR}}} \boldsymbol{z}_{v}\right\}_{v=-n(\mathrm{SNR})}^{-1}\right)}{\log \mathrm{SNR}} \geq r\left(1-P_{e}\right) .
$$

Since $-\log \operatorname{var}\left(\boldsymbol{h}_{0}+\frac{1}{\sqrt{\mathrm{SNR}}} \boldsymbol{z}_{0} \mid\left\{\boldsymbol{h}_{v}+\frac{1}{\sqrt{\mathrm{SNR}}} \boldsymbol{z}_{v}\right\}_{v=-n}^{-1}\right)$ is a monotone increasing function of $n$, it is easy to see 177 implicitly provides us with a lower bound on the scaling rate of $n(\mathrm{SNR})$.

In order to derive an explicit lower bound on the scaling rate of $n(\mathrm{SNR})$, we need to introduce a concept called transfinite diameter [12].

Definition 1: Let $\mathcal{S}$ be a compact set in the plane. Set

$$
\begin{aligned}
& V\left(z_{1}, \cdots, z_{n}\right)=\prod_{j>k}\left(z_{j}-z_{k}\right) \quad n \geq 2, z_{i} \in \mathcal{S} \\
& V_{n}(\mathcal{S})=\max _{z_{1}, \cdots, z_{n} \in \mathcal{S}}\left|V\left(z_{1}, \cdots, z_{n}\right)\right|
\end{aligned}
$$

and

$$
\tau_{n}(\mathcal{S})=\left[V_{n}(\mathcal{S})\right]^{\frac{2}{n(n-1)}}
$$

The transfinite diameter of $\mathcal{S}$ is defined by

$$
\tau(\mathcal{S})=\lim _{n \rightarrow \infty} \tau_{n}(\mathcal{S})
$$

We need the following facts regarding the transfinite diameter.

(i) For two compact sets $\mathcal{S}_{1}$ and $\mathcal{S}_{2}$ with $\mathcal{S}_{1} \subseteq \mathcal{S}_{2}$, we have $\tau\left(\mathcal{S}_{1}\right) \leq \tau\left(\mathcal{S}_{2}\right)$.

(ii) The diameter of the unit circle is 1 . More generally, the diameter of an arc of central angle $\theta$ on the unit circle is $\sin \left(\frac{\theta}{4}\right)$;

(iii) The transfinite diameter of any closed proper subset of the unit circle is less than 1 . 
A full discussion of the transfinite diameter can be found in [12].

Now return to the original problem. Since

$$
\operatorname{var}\left(\boldsymbol{h}_{0}+\frac{1}{\sqrt{\mathrm{SNR}}} \boldsymbol{z}_{0} \mid\left\{\boldsymbol{h}_{v}+\frac{1}{\sqrt{\mathrm{SNR}}} \boldsymbol{z}_{v}\right\}_{v=-n(\mathrm{SNR})}^{-1}\right) \geq \operatorname{var}\left(\boldsymbol{h}_{0} \mid\left\{\boldsymbol{h}_{v}\right\}_{v=-n(\mathrm{SNR})}^{-1}\right),
$$

we have

$$
\liminf _{\mathrm{SNR} \rightarrow \infty} \frac{-\log \operatorname{var}\left(\boldsymbol{h}_{0}+\frac{1}{\sqrt{\mathrm{SNR}}} \boldsymbol{z}_{0} \mid\left\{\boldsymbol{h}_{v}+\frac{1}{\sqrt{\mathrm{SNR}}} \boldsymbol{z}_{v}\right\}_{v=-n(\mathrm{SNR})}^{-1}\right)}{\log \mathrm{SNR}} \leq \liminf _{\mathrm{SNR} \rightarrow \infty} \frac{-\log \operatorname{var}\left(\boldsymbol{h}_{0} \mid\left\{\boldsymbol{h}_{v}\right\}_{v=-n(\mathrm{SNR})}^{-1}\right)}{\log \mathrm{SNR}} .
$$

Let $\mathcal{S}=\left\{e^{j \omega}: s\left(e^{j \omega}\right)>0\right\}$. It was shown in [13] that if the set $\mathcal{S}$ consists consist of a finite number of arcs of the unit circle, then

$$
\lim _{n \rightarrow \infty}\left(\operatorname{var}\left(\boldsymbol{h}_{0} \mid\left\{\boldsymbol{h}_{v}\right\}_{v=-n}^{-1}\right)\right)^{\frac{1}{n}}=\tau(\mathcal{S}) .
$$

Under the conditions

(a) The set $\mathcal{S}$ consists consist of a finite number of arcs of the unit circle,

(b) The set $\mathcal{S}$ is a closed proper subset of the unit circle,

it can be shown by using Facts (i), (ii) and (iii) that

$$
0<\tau(\mathcal{S})<1
$$

Therefore, under Conditions (a) and (b), we have

$$
\begin{aligned}
\liminf _{\mathrm{SNR} \rightarrow \infty} \frac{-\log \operatorname{var}\left(\boldsymbol{h}_{0} \mid\left\{\boldsymbol{h}_{v}\right\}_{v=-n(\mathrm{SNR})}^{-1}\right)}{\log \mathrm{SNR}} & =\liminf _{\mathrm{SNR} \rightarrow \infty} \frac{-\frac{1}{n(\mathrm{SNR})} \log \operatorname{var}\left(\boldsymbol{h}_{0} \mid\left\{\boldsymbol{h}_{v}\right\}_{v=-n(\mathrm{SNR})}^{-1}\right)}{\frac{1}{n(\mathrm{SNR})} \log \mathrm{SNR}} \\
& =\liminf _{\mathrm{SNR} \rightarrow \infty} \frac{-n(\mathrm{SNR}) \log \tau(\mathcal{S})}{\log \mathrm{SNR}} .
\end{aligned}
$$

It is clear that in order to guarantee that 18 is greater than or equal to $r\left(1-P_{e}\right)$, we must have

$$
\liminf _{\mathrm{SNR} \rightarrow \infty} \frac{n(\mathrm{SNR})}{\log \mathrm{SNR}} \geq-\frac{r\left(1-P_{e}\right)}{\log \tau(\mathrm{SNR})}
$$

which is a necessary condition on the scaling rate of $n(\mathrm{SNR})$.

In contrast, we show in Appendix VII and Appendix VIII that for the AWGN channel and memoryless coherent Rayleigh fading channel, it is possible to have the rate $R(\mathrm{SNR})$ grow linearly with log SNR with fixed codeword length $n$ and bounded decoding error probability at high SNR. For these two cases, to facilitate the calculation, we adopt the average power constraint. But our main conclusion holds also under the peak power constraint.

\section{CAPACITy PER Unit ENERgy}

In the preceding sections, we focused on the channel capacity in the high SNR regime. Now we proceed to characterize the behavior of channel capacity in the low average power regime for the block-stationary Gaussian fading channel model. To this end, we shall study the capacity per unit energy (which is denoted by $C_{p}(\mathrm{SNR})$ ) due to its intrinsic connection with the channel capacity in this regime. The following theorem provides a general expression for the capacity per unit energy. 
Theorem 3 ( [9], [14]):

$$
C_{p}(\mathrm{SNR})=\lim _{n \rightarrow \infty} \sup _{\boldsymbol{x}^{n} \in \mathbb{D}_{n}(\mathrm{SNR})} \frac{D\left(p_{\boldsymbol{y}^{n} \mid \boldsymbol{x}^{n}} \| D\left(p_{\boldsymbol{y}^{n} \mid 0^{n}}\right)\right.}{\left\|\boldsymbol{x}^{n}\right\|_{2}^{2}} .
$$

Furthermore, the capacity per unit energy is related to the capacity by

$$
C_{p}(\mathrm{SNR})=\sup _{\mathrm{P}>0} \frac{C(\mathrm{P}, \mathrm{SNR})}{\mathrm{P}}=\lim _{\mathrm{P} \rightarrow 0} \frac{C(\mathrm{P}, \mathrm{SNR})}{\mathrm{P}}
$$

where $C(\mathrm{P}, \mathrm{SNR})$ is the channel capacity with average power constraint $\mathrm{P}$ and peak power constraint SNR.

The following theorem is an extension of [14, Proposition 3.1] for the symbol-symbol stationary channel model to the block-stationary model.

Theorem 4: For the block-stationary Gaussian fading channel model given in (1),

$$
C_{p}(\mathrm{SNR})=1-\frac{1}{2 \pi \mathrm{SNR}} \min _{\mathcal{M} \subseteq\{1, \cdots, T\}} \Psi(\mathcal{M}, \mathrm{SNR})
$$

where

$$
\Psi(\mathcal{M}, \mathrm{SNR})=\frac{1}{|\mathcal{M}|} \int_{-\pi}^{\pi} \log \operatorname{det}\left[I_{|\mathcal{M}|}+\operatorname{SNR} S_{\mathcal{M}}\left(e^{j \omega}\right)\right] \mathrm{d} \omega,
$$

and $S_{\mathcal{M}}\left(e^{j \omega}\right)$ is an $|\mathcal{M}| \times|\mathcal{M}|$ principal minor of $S\left(e^{j \omega}\right)$ with the indices of columns and rows specified by $\mathcal{M}$.

Proof: The proof is omitted since it is almost identical to that for the symbol-by-symbol stationary fading channel [14]. The only difference is that although the capacity per unit energy of the block-stationary fading channel can be asymptotically achieved by temporal ON-OFF signaling, we have to determine how to allocate ON symbols in a block. It can be shown that the optimal allocation scheme is given by $\mathcal{M}^{*}$, which is the minimizer of $\min _{\mathcal{M} \in\{1, \cdots, T\}} \Psi(\mathcal{M})$. Here $\mathcal{M}^{*}$ might not be unique.

$C_{p}(\mathrm{SNR})$ is a monotonically increasing function of SNR. It is easy to see that $C_{p}(\mathrm{SNR})$ goes to 1 as $\mathrm{SNR} \rightarrow \infty$, and goes to 0 as SNR $\rightarrow 0$. The following result provides a more precise characterization of the convergence behavior.

Corollary 1: At high SNR,

$$
C_{p}(\mathrm{SNR})=1-\min _{\mathcal{M} \subseteq\{1, \cdots, T\}} \frac{\sum_{i=0}^{|\mathcal{M}|} i \mu\left(\operatorname{rank}\left(S_{\mathcal{M}}\left(e^{j \omega}\right)\right)=i\right) \log \mathrm{SNR}}{2 \pi|\mathcal{M}| \mathrm{SNR}}+o\left(\frac{\log \mathrm{SNR}}{\mathrm{SNR}}\right) .
$$

At low SNR, if

$$
\int_{-\pi}^{\pi} \operatorname{tr}\left[S^{2}\left(e^{j \omega}\right)\right] \mathrm{d} \omega<\infty
$$

then

Proof: By Lemma 1 at high SNR

$$
C_{p}(\mathrm{SNR})=\frac{\mathrm{SNR}}{4 \pi} \max _{\mathcal{M} \subseteq\{1, \cdots, T\}} \frac{1}{|\mathcal{M}|} \int_{-\pi}^{\pi} \operatorname{tr}\left[S_{\mathcal{M}}^{2}\left(e^{j \omega}\right)\right] \mathrm{d} \omega+o(\mathrm{SNR}) .
$$

$$
\int_{-\pi}^{\pi} \log \operatorname{det}\left[\frac{1}{\operatorname{SNR}} I_{|\mathcal{M}|}+S_{\mathcal{M}}\left(e^{j \omega}\right)\right] \mathrm{d} \omega=-\sum_{i=0}^{|\mathcal{M}|}(|\mathcal{M}|-i) \mu\left(\operatorname{rank}\left(S_{\mathcal{M}}\left(e^{j \omega}\right)\right)=i\right) \log \operatorname{SNR}+o(\log \mathrm{SNR}) .
$$


Therefore,

$$
\begin{aligned}
C_{p}(\mathrm{SNR}) & =1-\frac{1}{2 \pi \mathrm{SNR}} \min _{\mathcal{M} \subseteq\{1, \cdots, T\}} \Psi(\mathcal{M}, \mathrm{SNR}) \\
& =1-\frac{1}{2 \pi \mathrm{SNR}} \min _{\mathcal{M} \subseteq\{1, \cdots, T\}}\left\{\frac{1}{|\mathcal{M}|} \int_{-\pi}^{\pi} \log \operatorname{det}\left[\frac{1}{\mathrm{SNR}} I_{|\mathcal{M}|}+S_{\mathcal{M}}\left(e^{j \omega}\right)\right] \mathrm{d} \omega+2 \pi \log \mathrm{SNR}\right\} \\
& =1+\max _{\mathcal{M} \subseteq\{1, \cdots, T\}} \frac{\sum_{i=0}^{|\mathcal{M}|}(|\mathcal{M}|-i) \mu\left(\operatorname{rank}\left(S_{\mathcal{M}}\left(e^{j \omega}\right)\right)=i\right) \log \mathrm{SNR}}{2 \pi|\mathcal{M}| \mathrm{SNR}}-\frac{\log \mathrm{SNR}}{\mathrm{SNR}}+o\left(\frac{\log \mathrm{SNR}}{\mathrm{SNR}}\right) \\
& =1-\min _{\mathcal{M} \subseteq\{1, \cdots, T\}} \frac{\sum_{i=0}^{|\mathcal{M}|} i \mu\left(\operatorname{rank}\left(S_{\mathcal{M}}\left(e^{j \omega}\right)\right)=i\right) \log \mathrm{SNR}}{2 \pi|\mathcal{M}| \mathrm{SNR}}+o\left(\frac{\log \mathrm{SNR}}{\mathrm{SNR}}\right) .
\end{aligned}
$$

At low SNR, using the second-order approximation [15], we obtain

$$
\log \operatorname{det}\left[I_{|\mathcal{M}|}+\operatorname{SNR} S_{\mathcal{M}}\left(e^{j \omega}\right)\right]=\operatorname{tr}\left[S_{\mathcal{M}}\left(e^{j \omega}\right)\right] \mathrm{SNR}-\frac{1}{2} \operatorname{tr}\left[S_{\mathcal{M}}^{2}\left(e^{j \omega}\right)\right] \mathrm{SNR}^{2}+o\left(\mathrm{SNR}^{2}\right) .
$$

Therefore,

$$
\begin{aligned}
C_{p}(\mathrm{SNR}) & =1-\frac{1}{2 \pi \mathrm{SNR}} \min _{\mathcal{M} \subseteq\{1, \cdots, T\}} \Psi(\mathcal{M}, \mathrm{SNR}) \\
& =1-\frac{1}{2 \pi} \min _{\mathcal{M} \subseteq\{1, \cdots, T\}} \frac{1}{|\mathcal{M}|}\left\{\int_{-\pi}^{\pi} \operatorname{tr}\left[S_{\mathcal{M}}\left(e^{j \omega}\right)\right] \mathrm{d} \omega-\frac{1}{2} \int_{-\pi}^{\pi} \operatorname{tr}\left[S_{\mathcal{M}}^{2}\left(e^{j \omega}\right)\right] \mathrm{SNRd} \omega\right\}+o(\mathrm{SNR}) \\
& =\frac{\mathrm{SNR}}{4 \pi} \max _{\mathcal{M} \subseteq\{1, \cdots, T\}} \frac{1}{|\mathcal{M}|} \int_{-\pi}^{\pi} \operatorname{tr}\left[S_{\mathcal{M}}^{2}\left(e^{j \omega}\right)\right] \mathrm{d} \omega+o(\mathrm{SNR})
\end{aligned}
$$

where the last equality follows from the fact that

$$
\frac{1}{2 \pi|\mathcal{M}|} \int_{-\pi}^{\pi} \operatorname{tr}\left[S_{\mathcal{M}}\left(e^{j \omega}\right)\right] \mathrm{d} \omega=1
$$

Remark 6: Using the inequality

$$
\log \operatorname{det}\left[I_{|\mathcal{M}|}+\operatorname{SNR} S_{\mathcal{M}}\left(e^{j \omega}\right)\right] \geq \operatorname{tr}\left[S_{\mathcal{M}}\left(e^{j \omega}\right)\right] \operatorname{SNR}-\frac{1}{2} \operatorname{tr}\left[S_{\mathcal{M}}^{2}\left(e^{j \omega}\right)\right] \operatorname{SNR}{ }^{2},
$$

we can upper bound $C_{p}(\mathrm{SNR})$ by

$$
C_{p}(\mathrm{SNR}) \leq \frac{\mathrm{SNR}}{4 \pi} \max _{\mathcal{M} \subseteq\{1, \cdots, T\}} \frac{1}{|\mathcal{M}|} \int_{-\pi}^{\pi} \operatorname{tr}\left[S_{\mathcal{M}}^{2}\left(e^{j \omega}\right)\right] \mathrm{d} \omega .
$$

It can be seen from Corollary 1 that this upper bound is a good approximation of $C_{p}(\mathrm{SNR})$ in the low SNR regime.

Now we proceed to compute $C_{p}(\mathrm{SNR})$ in the following examples.

Example 5: When the channel changes independently from block to block, $C_{p}(\mathrm{SNR})$ is equal to

$$
1-\min _{\mathcal{M} \subseteq\{1, \cdots, T\}} \frac{1}{|\mathcal{M}| \mathrm{SNR}} \log \operatorname{det}\left[I_{|\mathcal{M}|}+\operatorname{SNR} R_{\mathcal{M}}(0)\right]
$$

where $R_{\mathcal{M}}(0)$ is an $|\mathcal{M}| \times|\mathcal{M}|$ principal minor of $R(0)$ with the indices of columns and rows specified by $\mathcal{M}$. If we further let the fading remain constant within a block, then all the entries of $R(0)$ are one. It is not difficult to show that

$$
\frac{1}{|\mathcal{M}| \mathrm{SNR}} \log \operatorname{det}\left[I_{|\mathcal{M}|}+\operatorname{SNR} R_{\mathcal{M}}(0)\right]=\frac{\log (1+|\mathcal{M}| \mathrm{SNR})}{|\mathcal{M}| \mathrm{SNR}}
$$


which is minimized when $|\mathcal{M}|=T$, i.e., $\mathcal{M}=\{1,2, \cdots, T\}$. So we have

$$
C_{p}(\mathrm{SNR})=1-\frac{\log (1+T \mathrm{SNR})}{T \mathrm{SNR}}
$$

as shown in [14].

Example 6: Consider the case in which the fading process satisfies the following conditions

1) All the off-diagonal entries of $R(0)$ are equal to $\alpha$, where $\alpha \in \mathbb{C}$ is a constant;

2) All the entries of $R(i)$ are equal to $\beta_{i}$ for any non-zero integer $i$, where $\beta_{i} \in \mathbb{C}$ is a constant that depends only on $i$.

We also know that the diagonal entries of $R(0)$ are all one. So for any fixed $\omega(-\pi \leq \omega \leq \pi)$, all the diagonal entries of $I+\operatorname{SNR} S\left(e^{j \omega}\right)$ are identical, and all the off-diagonal entries of $I+\operatorname{SNR} S\left(e^{j \omega}\right)$ are identical. It then follows from Szasz's inequality [16] that for any $\omega \in[-\pi, \pi]$,

$$
\left\{\operatorname{det}\left[I_{|\mathcal{M}|}+\operatorname{SNR} S_{\mathcal{M}}\left(e^{j \omega}\right)\right\}^{\frac{1}{|\mathcal{M}|}}\right.
$$

is minimized when $\mathcal{M}=\{1,2, \cdots, T\}$. In this case we therefor have

$$
C_{p}(\mathrm{SNR})=1-\frac{1}{2 \pi T \mathrm{SNR}} \int_{-\pi}^{\pi} \log \operatorname{det}\left(I+\operatorname{SNR} S\left(e^{j \omega}\right)\right) \mathrm{d} \omega .
$$

If the fading remains constant within a block, then for any fixed $\omega$, all the entries of $S\left(e^{j \omega}\right)$ are identical, which we shall denote by $s\left(e^{j \omega}\right)$. It can be shown that

$$
\operatorname{det}\left[I+\operatorname{SNR} S\left(e^{j \omega}\right)\right]=1+\operatorname{TSNR} s\left(e^{j \omega}\right)
$$

which yields

$$
C_{p}(\mathrm{SNR})=1-\frac{1}{2 \pi T \mathrm{SNR}} \int_{-\pi}^{\pi} \log \left[1+\operatorname{TSNR} s\left(e^{j \omega}\right)\right] \mathrm{d} \omega .
$$

We can see from 21 that $C_{p}(\mathrm{SNR})$ is a monotonically increasing function of $T$ and SNR. Intuitively, as $T$ gets larger and larger, the receiver can estimate the channel more and more accurately, and thus the capacity per unit energy of the non-coherent channel should converge to that of the coherent channel, which is equal to one; as SNR goes to infinity, $C_{p}(\mathrm{SNR})$ should also converge to one since flash signaling can be used if there is no peak power constraint (i.e., SNR $=\infty$ ) [17]. Moreover, (21) provides a precise characterization of the interplay between the coherence time and signal peakiness, stating that the capacity per unit energy is unaffected as long as the product of $T$ and SNR is fixed. See [18], [19] for a related discussion.

For the special case where the fading is a block Gauss-Markov process, i.e., all the entries of $R(i)$ are equal to $\rho^{i}$ if $i \geq 0$ and equal to $\left(\rho^{*}\right)^{-i}$ if $i<0$ for some $\rho \in \mathbb{C}$ with $0 \leq|\rho|<1$, we have

$$
1+\operatorname{TSNR} s\left(e^{j \omega}\right)=\left|\varphi\left(e^{j \omega}\right)\right|
$$

where

$$
\varphi(z)=\frac{\left(\rho^{*} z-\gamma_{0}\right)^{2}}{\gamma_{0}|\rho|^{2}\left(z-\frac{1}{\rho^{*}}\right)^{2}}
$$


and

$$
\gamma_{0}=\frac{b+\sqrt{b^{2}-4|\rho|^{2}}}{2}
$$

with $b=1+T S N R+|\rho|^{2}(1-T S N R)$. The function $\varphi$ is analytic and nonzero in a neighborhood of the unit disk. Thus, by Jensen's formula

$$
\begin{aligned}
C_{p}(\mathrm{SNR}) & =1-\frac{1}{2 \pi T \mathrm{SNR}} \int_{-\pi}^{\pi} \log \left|\varphi\left(e^{j \omega}\right)\right| \mathrm{d} \omega \\
& =1-\frac{1}{T \mathrm{SNR}} \log |\varphi(0)| \\
& =1-\frac{1}{T \mathrm{SNR}} \log \gamma_{0},
\end{aligned}
$$

from which we can recover [14, Corollary 4.1] by setting $T=1$.

Finding the optimal $\mathcal{M}^{*}$ is a difficult problem in general. Moreover, as shown in the following example, the optimal $\mathcal{M}^{*}$ may depend on the SNR level.

Example 7: Let the fading process be independent from block to block with

$$
S\left(e^{j \omega}\right)=R(0)=\left(\begin{array}{ccc}
1 & 1 & \rho^{*} \\
1 & 1 & \rho^{*} \\
\rho & \rho & 1
\end{array}\right)
$$

where $|\rho| \in[0,1]$.

It is shown in Appendix IX that

1) When $0 \leq|\rho| \leq \frac{1}{2}$, the optimal $\mathcal{M}^{*}$ is $\{1,2\}$, and

$$
C_{p}(\mathrm{SNR})=1-\frac{1}{2 \pi \mathrm{SNR}} \Psi(\{1,2\}, \mathrm{SNR})=1-\frac{\log (1+2 \mathrm{SNR})}{2 \mathrm{SNR}} ;
$$

2) When $\frac{1}{2}<|\rho|<1$,

$$
\mathcal{M}^{*}= \begin{cases}\{1,2,3\} & \text { SNR }<\frac{2|\rho|-1}{2(1-|\rho|)^{2}} \\ \{1,2\} \text { or }\{1,2,3\} & \text { SNR }=\frac{2|\rho|-1}{2(1-|\rho|)^{2}} \\ \{1,2\} & \text { SNR }>\frac{2|\rho|-1}{2(1-|\rho|)^{2}}\end{cases}
$$

and

$$
C_{p}(\mathrm{SNR})= \begin{cases}1-\frac{\log \left(1+3 \mathrm{SNR}+2 \mathrm{SNR}^{2}-2|\rho|^{2} \mathrm{SNR}^{2}\right)}{3 \mathrm{SNR}} & \mathrm{SNR}<\frac{2|\rho|-1}{2(1-|\rho|)^{2}} \\ 1-\frac{\log (1+2 \mathrm{SNR})}{2 \mathrm{SNR}} & \mathrm{SNR} \geq \frac{2|\rho|-1}{2(1-|\rho|)^{2}}\end{cases}
$$

3) When $|\rho|=1$, the optimal $\mathcal{M}^{*}$ is $\{1,2,3\}$, and

$$
C_{p}(\mathrm{SNR})=1-\frac{1}{2 \pi \mathrm{SNR}} \Psi(\{1,2,3\}, \mathrm{SNR})=1-\frac{\log (1+3 \mathrm{SNR})}{3 \mathrm{SNR}}
$$


It can be verified that this result is consistent with the asymptotic analysis in Corollary 1 Since $S_{\mathcal{M}}\left(e^{j \omega}\right)=$ $R_{\mathcal{M}}(0)$ for any $\mathcal{M} \subseteq\{1, \cdots, T\}$, it is easy to see that

$$
\sum_{i=0}^{|\mathcal{M}|} \frac{i}{|\mathcal{M}|} \mu\left(\operatorname{rank}\left(S_{\mathcal{M}}\left(e^{j \omega}\right)\right)=i\right)
$$

is minimized at $\mathcal{M}=\{1,2\}$ if $|\rho|<1$, and minimized at $\mathcal{M}=\{1,2,3\}$ if $|\rho|=1$. Therefore, by [19, the optimal $\mathcal{M}^{*}$ at high SNR should be $\{1,2\}$ if $|\rho|<1$, and should be $\{1,2,3\}$ if $|\rho|=1$. Since

$$
\begin{aligned}
& S_{\{1\}}^{2}\left(e^{j \omega}\right)=S_{\{2\}}^{2}\left(e^{j \omega}\right)=S_{\{3\}}^{2}\left(e^{j \omega}\right)=1, \\
& S_{\{1,3\}}^{2}\left(e^{j \omega}\right)=S_{\{2,3\}}^{2}\left(e^{j \omega}\right)=\left(\begin{array}{cc}
1+|\rho|^{2} & 2 \rho^{*} \\
2 \rho & 1+|\rho|^{2}
\end{array}\right), \\
& S_{\{1,2\}}^{2}\left(e^{j \omega}\right)=\left(\begin{array}{ll}
2 & 2 \\
2 & 2
\end{array}\right), \\
& S_{\{1,2,3\}}^{2}\left(e^{j \omega}\right)=\left(\begin{array}{ccc}
2+|\rho|^{2} & 2+|\rho|^{2} & 3 \rho^{*} \\
2+|\rho|^{2} & 2+|\rho|^{2} & 3 \rho^{2} \\
3 \rho & 3 \rho & 1+2|\rho|^{2}
\end{array}\right),
\end{aligned}
$$

it follows that $\frac{1}{|\mathcal{M}|} \operatorname{tr}\left[S_{\mathcal{M}}^{2}\left(e^{j \omega}\right)\right]$ is maximized at $\mathcal{M}=\{1,2\}$ if $|\rho|<\frac{1}{2}$, and maximized at $\mathcal{M}=\{1,2,3\}$ if $|\rho|>\frac{1}{2}$. Therefore, by (20), the optimal $\mathcal{M}^{*}$ at low SNR should be $\{1,2\}$ if $|\rho|<\frac{1}{2}$, and should be $\{1,2,3\}$ if $|\rho|>\frac{1}{2}$.

Intuitively, if $|\rho|$ is close to 1 , we can approximate $R(0)$ by the all-one matrix, and then it follows from Example 5 that the optimal $\mathcal{M}^{*}$ is $\{1,2,3\}$. The approximation breaks down at high SNR since Corollary 1 implies that the optimal $\mathcal{M}^{*}$ should be $\{1,2\}$ as $\mathrm{SNR} \rightarrow \infty$.

\section{CONCLUSION}

We conducted a detailed study of the block-stationary Gaussian fading channel model introduced in [7]. We derived single-letter upper and lower bounds on channel capacity, and used these bounds to characterize the asymptotic behavior of channel capacity. Specifically, we computed the asymptotic ratio between the non-coherent channel capacity and the logarithm of the SNR in the high SNR regime. This result generalizes many previous results on noncoherent capacity. We showed that the behavior of channel capacity depends critically on channel modelling. We also derived an expression for the capacity per unit energy for the block-stationary fading model. It is clearly of interest to generalize these results to the multi-antenna scenario, but such an extension seems technically nontrivial.

Another direction that we explored was the interplay between the codeword length, SNR level, and decoding error probability. We showed that for noncoherent symbol-by-symbol stationary fading channels, the codeword length must scale with SNR in order to guarantee that the communication rate can grow linearly with log SNR with bounded decoding error probability, and we found a necessary condition for the growth rate of the codeword length. 
We believe that a more complete characterization of the interplay between the codeword length, SNR level, and decoding error probability is of both theoretical significance and practical value.

\section{APPENDIX I}

\section{PROOF OF MONOTONICITY}

By the block stationarity of the fading process, we have

$I\left(\boldsymbol{x}_{i+j T} ; \boldsymbol{y}_{i+j T} \mid \mathbb{E}\left(\boldsymbol{h}_{i+j T} \mid\left\{\boldsymbol{h}_{k}+\frac{1}{x_{\min }} \boldsymbol{z}_{k}\right\}_{k=1}^{i+j T-1}\right)\right)=I\left(\boldsymbol{x}_{i} ; \boldsymbol{y}_{i} \mid \mathbb{E}\left(\boldsymbol{h}_{i} \mid\left\{\boldsymbol{h}_{k}+\frac{1}{x_{\min }} \boldsymbol{z}_{k}\right\}_{k=1-j T}^{i-1}\right)\right)$.

Since for any $j_{1}<j_{2}$,

$$
\boldsymbol{x}_{i} \rightarrow\left(\boldsymbol{y}_{i}, \mathbb{E}\left(\boldsymbol{h}_{i} \mid\left\{\boldsymbol{h}_{k}+\frac{1}{x_{\min }} \boldsymbol{z}_{k}\right\}_{k=1-j_{2} T}^{i-1}\right)\right) \rightarrow\left(\boldsymbol{y}_{i}, \mathbb{E}\left(\boldsymbol{h}_{i} \mid\left\{\boldsymbol{h}_{k}+\frac{1}{x_{\min }} \boldsymbol{z}_{k}\right\}_{k=1-j_{1} T}^{i-1}\right)\right)
$$

form a Markov chain, it follows by data processing inequality that

$$
I\left(\boldsymbol{x}_{i} ; \boldsymbol{y}_{i} \mid \mathbb{E}\left(\boldsymbol{h}_{i} \mid\left\{\boldsymbol{h}_{k}+\frac{1}{x_{\min }} \boldsymbol{z}_{k}\right\}_{k=1-j_{1} T}^{i-1}\right)\right) \leq I\left(\boldsymbol{x}_{i} ; \boldsymbol{y}_{i} \mid \mathbb{E}\left(\boldsymbol{h}_{i} \mid\left\{\boldsymbol{h}_{k}+\frac{1}{x_{\min }} \boldsymbol{z}_{k}\right\}_{k=1-j_{2} T}^{i-1}\right)\right) \text {. }
$$

Equations (22) and (23) together imply that $\left\{I\left(\boldsymbol{x}_{i+j T} ; \boldsymbol{y}_{i+j T} \mid \mathbb{E}\left(\boldsymbol{h}_{i+j T} \mid\left\{\boldsymbol{h}_{k}+\frac{1}{x_{\min }} \boldsymbol{z}_{k}\right\}_{k=1}^{i+j T-1}\right)\right)\right\}_{j}$ is a monotone increasing sequence.

For every $\mathbb{E}\left(\boldsymbol{h}_{i} \mid\left\{\boldsymbol{h}_{k}+\frac{1}{x_{\min }} \boldsymbol{z}_{k}\right\}_{k=1-j T}^{i-1}\right)$, we can construct a random variable $\boldsymbol{\Delta}_{j} \sim \mathcal{C N}\left(0, \delta_{j}\right)$ independent of everything else such that

$$
\mathbb{E}\left(\boldsymbol{h}_{i} \mid\left\{\boldsymbol{h}_{k}+\frac{1}{x_{\min }} \boldsymbol{z}_{k}\right\}_{k=1-j T}^{i-1}\right)=\mathbb{E}\left(\boldsymbol{h}_{i} \mid \mathbb{E}\left(\boldsymbol{h}_{i} \mid\left\{\boldsymbol{h}_{k}+\frac{1}{x_{\min }} \boldsymbol{z}_{k}\right\}_{k=-\infty}^{i-1}\right)+\Delta_{j}\right)
$$

in distribution. Clearly, $\delta_{j} \rightarrow 0$ as $j \rightarrow \infty$. Moreover, it is not difficult to show that

$$
I\left(\boldsymbol{x}_{i} ; \boldsymbol{y}_{i} \mid \mathbb{E}\left(\boldsymbol{h}_{i} \mid\left\{\boldsymbol{h}_{k}+\frac{1}{x_{\min }} \boldsymbol{z}_{k}\right\}_{k=1-j T}^{i-1}\right)\right)=I\left(\boldsymbol{x}_{i} ; \boldsymbol{y}_{i} \mid \mathbb{E}\left(\boldsymbol{h}_{i} \mid\left\{\boldsymbol{h}_{k}+\frac{1}{x_{\min }} \boldsymbol{z}_{k}\right\}_{k=-\infty}^{i-1}\right)+\Delta_{j}\right) .
$$

Combining (22) and (24), we get

$$
\begin{aligned}
& \lim _{j \rightarrow \infty} I\left(\boldsymbol{x}_{i+j T} ; \boldsymbol{y}_{i+j T} \mid \mathbb{E}\left(\boldsymbol{h}_{i+j T} \mid\left\{\boldsymbol{h}_{k}+\frac{1}{x_{\min }} \boldsymbol{z}_{k}\right\}_{k=1}^{i+j T-1}\right)\right) \\
= & \lim _{j \rightarrow \infty} I\left(\boldsymbol{x}_{i} ; \boldsymbol{y}_{i} \mid \mathbb{E}\left(\boldsymbol{h}_{i} \mid\left\{\boldsymbol{h}_{k}+\frac{1}{x_{\min }} \boldsymbol{z}_{k}\right\}_{k=1-j T}^{i-1}\right)\right) \\
= & \lim _{j \rightarrow \infty} I\left(\boldsymbol{x}_{i} ; \boldsymbol{y}_{i} \mid \mathbb{E}\left(\boldsymbol{h}_{i} \mid\left\{\boldsymbol{h}_{k}+\frac{1}{x_{\min }} \boldsymbol{z}_{k}\right\}_{k=-\infty}^{i-1}\right)+\Delta_{j}\right) .
\end{aligned}
$$

Since

$$
\begin{aligned}
& I\left(\boldsymbol{x}_{i} ; \boldsymbol{y}_{i} \mid \mathbb{E}\left(\boldsymbol{h}_{i} \mid\left\{\boldsymbol{h}_{k}+\frac{1}{x_{\min }} \boldsymbol{z}_{k}\right\}_{k=-\infty}^{i-1}\right)+\Delta_{j}\right) \\
= & I\left(\boldsymbol{x}_{i} ; \boldsymbol{y}_{i}, \mathbb{E}\left(\boldsymbol{h}_{i} \mid\left\{\boldsymbol{h}_{k}+\frac{1}{x_{\min }} \boldsymbol{z}_{k}\right\}_{k=-\infty}^{i-1}\right)+\Delta_{j}\right) \\
= & h\left(\boldsymbol{y}_{i}, \mathbb{E}\left(\boldsymbol{h}_{i} \mid\left\{\boldsymbol{h}_{k}+\frac{1}{x_{\min }} \boldsymbol{z}_{k}\right\}_{k=-\infty}^{i-1}\right)+\Delta_{j}\right)-h\left(\boldsymbol{y}_{i}, \mathbb{E}\left(\boldsymbol{h}_{i} \mid\left\{\boldsymbol{h}_{k}+\frac{1}{x_{\min }} \boldsymbol{z}_{k}\right\}_{k=-\infty}^{i-1}\right)+\Delta_{j} \mid \boldsymbol{x}_{i}\right) .
\end{aligned}
$$


By [3, Lemma 6.11], we get

$$
\lim _{j \rightarrow \infty} h\left(\boldsymbol{y}_{i}, \mathbb{E}\left(\boldsymbol{h}_{i} \mid\left\{\boldsymbol{h}_{k}+\frac{1}{x_{\min }} \boldsymbol{z}_{k}\right\}_{k=-\infty}^{i-1}\right)+\Delta_{j}\right)=h\left(\boldsymbol{y}_{i}, \mathbb{E}\left(\boldsymbol{h}_{i} \mid\left\{\boldsymbol{h}_{k}+\frac{1}{x_{\min }} \boldsymbol{z}_{k}\right\}_{k=-\infty}^{i-1}\right)\right) .
$$

Since conditioned on $\boldsymbol{x}_{i},\left(\boldsymbol{y}_{i}, \mathbb{E}\left(\boldsymbol{h}_{i} \mid\left\{\boldsymbol{h}_{k}+\frac{1}{x_{\min }} \boldsymbol{z}_{k}\right\}_{k=-\infty}^{i-1}\right)+\Delta_{j}\right)$ are jointly Gaussian with uniformly bounded differential entropy for any realization of $\boldsymbol{x}_{i}$ (Note: $\left|\boldsymbol{x}_{i}\right|^{2} \leq \mathrm{SNR}$ ), it follows by dominated convergence theorem that

$$
\lim _{j \rightarrow \infty} h\left(\boldsymbol{y}_{i}, \mathbb{E}\left(\boldsymbol{h}_{i} \mid\left\{\boldsymbol{h}_{k}+\frac{1}{x_{\min }} \boldsymbol{z}_{k}\right\}_{k=-\infty}^{i-1}\right)+\Delta_{j} \mid \boldsymbol{x}_{i}\right)=h\left(\boldsymbol{y}_{i}, \mathbb{E}\left(\boldsymbol{h}_{i} \mid\left\{\boldsymbol{h}_{k}+\frac{1}{x_{\min }} \boldsymbol{z}_{k}\right\}_{k=-\infty}^{i-1}\right) \mid \boldsymbol{x}_{i}\right) .
$$

Therefore,

$\lim _{j \rightarrow \infty} I\left(\boldsymbol{x}_{i+j T} ; \boldsymbol{y}_{i+j T} \mid \mathbb{E}\left(\boldsymbol{h}_{i+j T} \mid\left\{\boldsymbol{h}_{k}+\frac{1}{x_{\min }} \boldsymbol{z}_{k}\right\}_{k=1}^{i+j T-1}\right)\right)=\lim _{j \rightarrow \infty} I\left(\boldsymbol{x}_{i} ; \boldsymbol{y}_{i} \mid \mathbb{E}\left(\boldsymbol{h}_{i} \mid\left\{\boldsymbol{h}_{k}+\frac{1}{x_{\min }} \boldsymbol{z}_{k}\right\}_{k=-\infty}^{i-1}\right)\right)$.

\section{APPENDIX II}

\section{ProOF OF LEMMA 1}

By eigenvalue decomposition, we write

$$
A(\xi)=U(\xi) D(\xi) U^{\dagger}(\xi)
$$

and

$$
A(\xi)+\epsilon I_{M}=U(\xi)\left(D(\xi)+\epsilon I_{M}\right) U^{\dagger}(\xi)
$$

where $U(\xi)$ is a unitary matrix, and $D(\xi)$ is a diagonal matrix with nonnegative diagonal entries. Since $\operatorname{rank}(A(\xi))=$ $\operatorname{rank}(D(\xi))$, define

$$
\Omega_{i}=\{\xi: \operatorname{rank}(A(\xi))=\operatorname{rank}(D(\xi))=i\}, \quad 0 \leq i \leq M .
$$

We have

$$
\begin{aligned}
\lim _{\epsilon \rightarrow 0} \frac{\int_{\xi_{0}}^{\xi_{1}} \log \operatorname{det}\left[A(\xi)+\epsilon I_{M}\right] \mathrm{d} \xi}{\log \epsilon} & =\lim _{\epsilon \rightarrow 0} \frac{\int_{\xi_{1}}^{\xi_{2}} \log \operatorname{det}\left[D(\xi)+\epsilon I_{M}\right] \mathrm{d} \xi}{\log \epsilon} \\
& =\lim _{\epsilon \rightarrow 0} \frac{-\sum_{i=0}^{M} \int_{\Omega_{i}} \log \operatorname{det}\left[D(\xi)+\epsilon I_{M}\right] \mathrm{d} \xi}{\log \epsilon} .
\end{aligned}
$$

For $\xi \in \Omega_{i}$, (possibly after permutating diagonal entries) we can write $D(\xi)=\operatorname{diag}\left\{d_{1}(\xi), \cdots, d_{i}(\xi), 0, \cdots, 0\right\}$, where $d_{j}(\xi)>0,1 \leq j \leq i$. Therefore,

$$
\lim _{\epsilon \rightarrow 0} \frac{\int_{\xi_{0}}^{\xi_{1}} \log \operatorname{det}\left[A(\xi)+\epsilon I_{M}\right] \mathrm{d} \xi}{\log \epsilon}=\lim _{\epsilon \rightarrow 0} \frac{-\sum_{i=0}^{M} \int_{\Omega_{i}} \sum_{j=1}^{i} \log \left[d_{j}(\xi)+\epsilon\right] \mathrm{d} \xi}{\log \epsilon}+\sum_{i=0}^{M}(M-i) \mu(\operatorname{rank}(A(\xi))=i)
$$

where $\mu(\operatorname{rank}(A(\xi))=i)$ is the Lebesgue measure of $\Omega_{i}$. By the argument in [6, Section VIII], it can be shown that

$$
\lim _{\epsilon \rightarrow 0} \frac{\int_{\Omega_{i}} \log \left[d_{i}(\xi)+\epsilon\right] \mathrm{d} \xi}{\log \epsilon}=0 .
$$


So we have

$$
\lim _{\epsilon \rightarrow 0} \frac{\int_{\xi_{0}}^{\xi_{1}} \log \operatorname{det}[A(\xi)+\epsilon I] \mathrm{d} \xi}{\log \epsilon}=\sum_{i=0}^{M}(M-i) \mu(\operatorname{rank}(A(\xi))=i)
$$

APPENDIX III

PROOF OF THEOREM 1

Define

$$
\sigma_{i}(\mathrm{SNR})=\operatorname{var}\left(\boldsymbol{h}_{i}+\frac{1}{\sqrt{\mathrm{SNR}}} \boldsymbol{z}_{i} \mid\left\{\boldsymbol{h}_{k}+\frac{1}{\sqrt{\mathrm{SNR}}} \boldsymbol{z}_{k}\right\}_{k=-\infty}^{i-1}\right) \quad i=1,2, \cdots, T .
$$

In the lower bound (5), let $\boldsymbol{x}_{i}$ be uniformly distributed over the set $\left\{z \in \mathbb{C}: \frac{\sqrt{\mathrm{SNR}}}{2} \leq\|z\| \leq \sqrt{\mathrm{SNR}}\right\}$. By Lemma 2

$$
\begin{aligned}
& I\left(\boldsymbol{x}_{i} ; \boldsymbol{h}_{i} \boldsymbol{x}_{i}+\boldsymbol{z}_{i} \mid \mathbb{E}\left(\boldsymbol{h}_{i} \mid\left\{\boldsymbol{h}_{k}+\frac{4}{\mathrm{SNR}} \boldsymbol{z}_{k}\right\}_{k=-\infty}^{i-1}\right)\right) \\
\geq & -\log \left[\sigma_{i}\left(\frac{\mathrm{SNR}}{4}\right)-\frac{12}{5 \mathrm{SNR}}\right]+\log \left(1-\sigma_{i}\left(\frac{\mathrm{SNR}}{4}\right)+\frac{4}{\mathrm{SNR}}\right)-\gamma-\log \frac{5 e}{6} \\
= & -\log \left[\sigma_{i}\left(\frac{\mathrm{SNR}}{4}\right)-\frac{12}{5 \mathrm{SNR}}\right]+o(\log \mathrm{SNR}) .
\end{aligned}
$$

Since $\sigma_{i}\left(\frac{\mathrm{SNR}}{4}\right) \geq \frac{4}{\mathrm{SNR}}$, it follows that

$$
\liminf _{\mathrm{SNR} \rightarrow \infty} \frac{I\left(\boldsymbol{x}_{i} ; \boldsymbol{h}_{i} \boldsymbol{x}_{i}+\boldsymbol{z}_{i} \mid \mathbb{E}\left(\boldsymbol{h}_{i} \mid\left\{\boldsymbol{h}_{k}+\frac{4}{\mathrm{SNR}} \boldsymbol{z}_{k}\right\}_{k=-\infty}^{i-1}\right)\right)}{\log \mathrm{SNR}} \geq \liminf _{\mathrm{SNR} \rightarrow \infty} \frac{-\log \left[\sigma_{i}\left(\frac{\mathrm{SNR}}{4}\right)\right]}{\log \mathrm{SNR}} .
$$

Let $\Sigma\left(\frac{\mathrm{SNR}}{4}\right)=L\left(\frac{\mathrm{SNR}}{4}\right) \Lambda\left(\frac{\mathrm{SNR}}{4}\right) L^{\dagger}\left(\frac{\mathrm{SNR}}{4}\right)$, where $L\left(\frac{\mathrm{SNR}}{4}\right)$ is a lower triangular matrix with unit diagonal entries, and $\Lambda\left(\frac{\mathrm{SNR}}{4}\right)=\operatorname{diag}\left\{\sigma_{1}\left(\frac{\mathrm{SNR}}{4}\right), \sigma_{2}\left(\frac{\mathrm{SNR}}{4}\right), \cdots, \sigma_{T}\left(\frac{\mathrm{SNR}}{4}\right)\right\}$. We have

$$
\begin{aligned}
\operatorname{det}\left[\Sigma\left(\frac{\mathrm{SNR}}{4}\right)\right] & =\operatorname{det}\left[L\left(\frac{\mathrm{SNR}}{4}\right)\right] \operatorname{det}\left[\Lambda\left(\frac{\mathrm{SNR}}{4}\right)\right] \operatorname{det}\left[L^{\dagger}\left(\frac{\mathrm{SNR}}{4}\right)\right] \\
& =\operatorname{det}\left[\Lambda\left(\frac{\mathrm{SNR}}{4}\right)\right] \\
& =\prod_{i=1}^{T} \sigma_{i}\left(\frac{\mathrm{SNR}}{4}\right) .
\end{aligned}
$$

Therefore,

$$
\begin{aligned}
\liminf _{\mathrm{SNR} \rightarrow \infty} \frac{C(\mathrm{SNR})}{\log \mathrm{SNR}} & \geq \liminf _{\mathrm{SNR} \rightarrow \infty} \frac{\sum_{i=1}^{T} I\left(\boldsymbol{x}_{i} ; \boldsymbol{h}_{i} \boldsymbol{x}_{i}+\boldsymbol{z}_{i} \mid \mathbb{E}\left(\boldsymbol{h}_{i} \mid\left\{\boldsymbol{h}_{k}+\frac{4}{\mathrm{SNR}} \boldsymbol{z}_{k}\right\}_{k=-\infty}^{i-1}\right)\right)}{T \log \mathrm{SNR}} \\
& \geq \liminf _{\mathrm{SNR} \rightarrow \infty} \frac{-\log \left[\prod_{i=1}^{T} \sigma_{i}\left(\frac{\mathrm{SNR}}{4}\right)\right]}{\log \mathrm{SNR}} \\
& =\liminf _{\mathrm{SNR} \rightarrow \infty} \frac{-\log \operatorname{det}\left[\Sigma\left(\frac{\mathrm{SNR}}{4}\right)\right]}{\log \mathrm{SNR}} \\
& =\liminf _{\mathrm{SNR} \rightarrow \infty} \frac{-\log \operatorname{det}[\Sigma(\mathrm{SNR})]}{\log \mathrm{SNR}}
\end{aligned}
$$


We use (8) derive an upper bound on $\frac{\log C(\text { SNR })}{\log \text { SNR }}$. First it is easy to see that

$$
\begin{aligned}
& \sup _{P_{\boldsymbol{x}_{k}} \in \mathcal{P}_{1}(\mathrm{SNR})} I\left(\boldsymbol{x}_{k}, \mathbb{E}\left(\boldsymbol{h}_{k} \mid\left\{\boldsymbol{h}_{t}+\frac{1}{\sqrt{\mathrm{SNR}}} \boldsymbol{z}_{t}\right\}_{t=-\infty}^{k-1}\right) ; \boldsymbol{y}_{k}\right) \\
\leq & \sup _{P_{\boldsymbol{x}_{k}} \in \mathcal{P}_{1}(\mathrm{SNR})} I\left(\mathbb{E}\left(\boldsymbol{h}_{k} \mid\left\{\boldsymbol{h}_{t}+\frac{1}{\sqrt{\mathrm{SNR}}} \boldsymbol{z}_{t}\right\}_{t=-\infty}^{k-1}\right) ; \boldsymbol{y}_{k} \mid \boldsymbol{x}_{k}\right)+\sup _{P_{\boldsymbol{x}_{k}} \in \mathcal{P}_{1}(\mathrm{SNR})} I\left(\boldsymbol{x}_{k} ; \boldsymbol{y}_{k}\right) .
\end{aligned}
$$

It is shown in [3] that

$$
\sup _{P_{\boldsymbol{x}_{k}} \in \mathcal{P}_{1}(\mathrm{SNR})} I\left(\boldsymbol{x}_{k} ; \boldsymbol{y}_{k}\right)=o(\log \mathrm{SNR}) .
$$

Now we proceed to upper-bound the first term in 27.

$$
\begin{aligned}
& \sup _{P_{\boldsymbol{x}_{k}} \in \mathcal{P}_{1}(\mathrm{SNR})} I\left(\mathbb{E}\left(\boldsymbol{h}_{k} \mid\left\{\boldsymbol{h}_{t}+\frac{1}{\sqrt{\mathrm{SNR}}} \boldsymbol{z}_{t}\right\}_{t=-\infty}^{k-1}\right) ; \boldsymbol{y}_{k} \mid \boldsymbol{x}_{k}\right) \\
= & \sup _{P_{\boldsymbol{x}_{k}} \in \mathcal{P}_{1}(\mathrm{SNR})} \mathbb{E}\left\{\log \left[\frac{1+\left|\boldsymbol{x}_{k}\right|^{2}}{1+\left|\boldsymbol{x}_{k}\right|^{2} \cdot \operatorname{var}\left(\boldsymbol{h}_{k} \mid\left\{\boldsymbol{h}_{t}+\frac{1}{\sqrt{\mathrm{SNR}}} \boldsymbol{z}_{t}\right\}_{t=-\infty}^{k-1}\right)}\right]\right\} \\
\leq & \log \left[\frac{1+\mathrm{SNR}}{1+\mathrm{SNR} \cdot \operatorname{var}\left(\boldsymbol{h}_{k} \mid\left\{\boldsymbol{h}_{t}+\frac{1}{\sqrt{\mathrm{SNR}}} \boldsymbol{z}_{t}\right\}_{t=-\infty}^{k-1}\right)}\right] \\
= & \log \left[\frac{1+\mathrm{SNR}}{\mathrm{SNR} \cdot \sigma_{k}(\mathrm{SNR})}\right] \cdot
\end{aligned}
$$

Therefore,

$$
\begin{aligned}
\limsup _{\mathrm{SNR} \rightarrow \infty} \frac{C(\mathrm{SNR})}{\log \mathrm{SNR}} & \leq \limsup _{\mathrm{SNR} \rightarrow \infty} \frac{\sum_{k=1}^{T} \sup _{\boldsymbol{P}_{k} \in \mathcal{P}_{1}(\mathrm{SNR})} I\left(\mathbb{E}\left(\boldsymbol{h}_{k} \mid\left\{\boldsymbol{h}_{t}+\frac{1}{\sqrt{\mathrm{SNR}}} \boldsymbol{z}_{t}\right\}_{t=-\infty}^{k-1}\right) ; \boldsymbol{y}_{k} \mid \boldsymbol{x}_{k}\right)}{T \log \mathrm{SNR}} \\
& \leq \limsup _{\mathrm{SNR} \rightarrow \infty} \frac{-\log \left[\prod_{k=1}^{T} \sigma_{k}(\mathrm{SNR})\right]}{\log \mathrm{SNR}} \\
& =\limsup _{\mathrm{SNR} \rightarrow \infty} \frac{-\log \operatorname{det}[\Sigma(\mathrm{SNR})]}{\log \mathrm{SNR}} .
\end{aligned}
$$

The desired result follows by combining (26) and 28.

\section{APPENDIX IV}

PROOF OF THEOREM 2

Define

$$
\sigma_{i}(\infty)=\operatorname{var}\left(\boldsymbol{h}_{i} \mid\left\{\boldsymbol{h}_{k}\right\}_{k=-\infty}^{i-1}\right) \quad i=1,2, \cdots, T .
$$

Similar to 25, we have

$$
\operatorname{det}[\Sigma(\infty)]=\prod_{i=1}^{T} \sigma_{i}(\infty)
$$


Therefore, $\operatorname{det}[\Sigma(\infty)]>0$ implies $\sigma_{i}(\infty)>0$ for all $i$.

Note that if $x_{\min }>\delta$ for some $\delta>0$, then

$$
\boldsymbol{x}_{i} \rightarrow\left(\boldsymbol{y}_{i}, \mathbb{E}\left(\boldsymbol{h}_{i} \mid\left\{\boldsymbol{h}_{k}+\frac{1}{x_{\min }} \boldsymbol{z}_{k}\right\}_{k=-\infty}^{i-1}\right)\right) \rightarrow\left(\boldsymbol{y}_{i}, \mathbb{E}\left(\boldsymbol{h}_{i} \mid\left\{\boldsymbol{h}_{k}+\delta \boldsymbol{z}_{k}\right\}_{k=-\infty}^{i-1}\right)\right)
$$

form a Markov chain, and

$$
I\left(\boldsymbol{x}_{i} ; \boldsymbol{h}_{i} \boldsymbol{x}_{i}+\boldsymbol{z}_{i} \mid \mathbb{E}\left(\boldsymbol{h}_{i} \mid\left\{\boldsymbol{h}_{k}+\frac{1}{x_{\min }} \boldsymbol{z}_{k}\right\}_{k=-\infty}^{i-1}\right)\right) \geq I\left(\boldsymbol{x}_{i} ; \boldsymbol{h}_{i} \boldsymbol{x}_{i}+\boldsymbol{z}_{i} \mid \mathbb{E}\left(\boldsymbol{h}_{i} \mid\left\{\boldsymbol{h}_{k}+\delta \boldsymbol{z}_{k}\right\}_{k=-\infty}^{i-1}\right)\right) .
$$

In the lower bound [5], let $\log \left|\boldsymbol{x}_{i}\right|^{2}$ be uniformly distributed over the interval [ $\left.\log x_{\min }^{2}, \log \mathrm{SNR}\right]$. As $\log x_{\min }^{2}$ grows sublinearly in $\log$ SNR to infinity, we get

$$
\begin{aligned}
& \liminf _{\mathrm{SNR} \rightarrow \infty}\left[I\left(\boldsymbol{x}_{i} ; \boldsymbol{h}_{i} \boldsymbol{x}_{i}+\boldsymbol{z}_{i} \mid \mathbb{E}\left(\boldsymbol{h}_{i} \mid\left\{\boldsymbol{h}_{k}+\frac{1}{x_{\min }} \boldsymbol{z}_{k}\right\}_{k=-\infty}^{i-1}\right)\right)-\log \log \mathrm{SNR}\right] \\
\geq & \liminf _{\mathrm{SNR} \rightarrow \infty}\left[I\left(\boldsymbol{x}_{i} ; \boldsymbol{h}_{i} \boldsymbol{x}_{i}+\boldsymbol{z}_{i} \mid \mathbb{E}\left(\boldsymbol{h}_{i} \mid\left\{\boldsymbol{h}_{k}+\delta \boldsymbol{z}_{k}\right\}_{k=-\infty}^{i-1}\right)\right)-\log \log \mathrm{SNR}\right] \\
= & -1-\gamma-\log \operatorname{var}\left(\boldsymbol{h}_{i} \mid\left\{\boldsymbol{h}_{j}+\delta \boldsymbol{z}_{j}\right\}_{j=-\infty}^{i-1}\right)
\end{aligned}
$$

where the last equality follows from [3, Proposition 4.23]. Therefore,

$$
\begin{aligned}
& \liminf _{\mathrm{SNR} \rightarrow \infty}[C(\mathrm{SNR})-\log \log \mathrm{SNR}] \\
\geq & \liminf _{\mathrm{SNR} \rightarrow \infty}\left[\frac{1}{T} \sum_{i=1}^{T} I\left(\boldsymbol{x}_{i} ; \boldsymbol{h}_{i} \boldsymbol{x}_{i}+\boldsymbol{z}_{i} \mid \mathbb{E}\left(\boldsymbol{h}_{i} \mid\left\{\boldsymbol{h}_{k}+\frac{1}{x_{\min }} \boldsymbol{z}_{k}\right\}_{k=-\infty}^{i-1}\right)\right)-\log \log \mathrm{SNR}\right] \\
= & -1-\gamma-\frac{1}{T} \sum_{i=1}^{T} \log \operatorname{var}\left(\boldsymbol{h}_{i} \mid\left\{\boldsymbol{h}_{j}+\delta \boldsymbol{z}_{j}\right\}_{j=-\infty}^{i-1}\right) .
\end{aligned}
$$

Since (29) holds for arbitrary positive $\delta$, it follows that

$$
\begin{aligned}
\liminf _{\mathrm{SNR} \rightarrow \infty}[C(\mathrm{SNR})-\log \log \mathrm{SNR}] & \geq-1-\gamma-\frac{1}{T} \lim _{\delta \rightarrow 0} \sum_{i=1}^{T} \log \operatorname{var}\left(\boldsymbol{h}_{i} \mid\left\{\boldsymbol{h}_{j}+\delta \boldsymbol{z}_{j}\right\}_{j=-\infty}^{i-1}\right) \\
& =-1-\gamma-\frac{1}{T} \log \operatorname{det}[\Sigma(\infty)] .
\end{aligned}
$$

From the upper bound $(8)$, we have

$$
\begin{aligned}
& \limsup _{\mathrm{SNR} \rightarrow \infty}[C(\mathrm{SNR})-\log \log \mathrm{SNR}] \\
\leq & \limsup _{\mathrm{SNR} \rightarrow \infty}\left[\frac{1}{T} \sum_{k=1}^{T} \sup _{P_{\boldsymbol{x}_{k}} \in \mathcal{P}_{1}(\mathrm{SNR})} I\left(\boldsymbol{x}_{k}, \mathbb{E}\left(\boldsymbol{h}_{k} \mid\left\{\boldsymbol{h}_{t}+\frac{1}{\sqrt{\mathrm{SNR}}} \boldsymbol{z}_{t}\right\}_{t=-\infty}^{k-1}\right) ; \boldsymbol{y}_{k}\right)-\log \log \mathrm{SNR}\right] \\
\leq & \limsup _{\mathrm{SNR} \rightarrow \infty}\left[\frac{1}{T} \sum_{k=1}^{T} \sup _{P_{\boldsymbol{x}_{k}} \in \mathcal{P}_{1}(\mathrm{SNR})} I\left(\boldsymbol{x}_{k}, \mathbb{E}\left(\boldsymbol{h}_{k} \mid\left\{\boldsymbol{h}_{j}\right\}_{j=-\infty}^{k-1}\right) ; \boldsymbol{y}_{k}\right)-\log \log \mathrm{SNR}\right] \\
\leq & \limsup _{\mathrm{SNR} \rightarrow \infty}\left[\sup _{P_{\boldsymbol{x}_{k}} \in \mathcal{P}_{1}(\mathrm{SNR})} I\left(\boldsymbol{x}_{1} ; \boldsymbol{y}_{1}\right)+\frac{1}{T} \sum_{k=1}^{T} \sup _{P_{\boldsymbol{x}_{k}} \in \mathcal{P}_{1}(\mathrm{SNR})} I\left(\mathbb{E}\left(\boldsymbol{h}_{k} \mid\left\{\boldsymbol{h}_{j}\right\}_{j=-\infty}^{k-1}\right) ; \boldsymbol{y}_{k} \mid \boldsymbol{x}_{k}\right)-\log \log \mathrm{SNR}\right]
\end{aligned}
$$

where (31) follows from the fact that

$$
\left(\boldsymbol{x}_{k}, \mathbb{E}\left(\boldsymbol{h}_{k} \mid\left\{\boldsymbol{h}_{t}+\frac{1}{\sqrt{\mathrm{SNR}}} \boldsymbol{z}_{t}\right\}_{t=-\infty}^{k-1}\right)\right) \rightarrow\left(\boldsymbol{x}_{k}, \mathbb{E}\left(\boldsymbol{h}_{k} \mid\left\{\boldsymbol{h}_{j}\right\}_{j=-\infty}^{k-1}\right)\right) \rightarrow \boldsymbol{y}_{k}
$$


form a Markov chain.

It was shown in [3, Corollary 4.19] that

$$
\lim _{\mathrm{SNR} \rightarrow \infty}\left[\sup _{P_{\boldsymbol{x}_{k}} \in \mathcal{P}_{1}(\mathrm{SNR})} I\left(\boldsymbol{x}_{1} ; \boldsymbol{y}_{1}\right)-\log \log \mathrm{SNR}\right]=-1-\gamma .
$$

Furthermore,

$$
\begin{aligned}
I\left(\mathbb{E}\left(\boldsymbol{h}_{k} \mid\left\{\boldsymbol{h}_{j}\right\}_{j=-\infty}^{k-1}\right) ; \boldsymbol{y}_{k} \mid \boldsymbol{x}_{k}\right) & \leq I\left(\mathbb{E}\left(\boldsymbol{h}_{k} \mid\left\{\boldsymbol{h}_{j}\right\}_{j=-\infty}^{k-1}\right) ; \boldsymbol{h}_{k}, \boldsymbol{y}_{k} \mid \boldsymbol{x}_{k}\right) \\
& =I\left(\mathbb{E}\left(\boldsymbol{h}_{k} \mid\left\{\boldsymbol{h}_{j}\right\}_{j=-\infty}^{k-1}\right) ; \boldsymbol{h}_{k}\right)+I\left(\mathbb{E}\left(\boldsymbol{h}_{k} \mid\left\{\boldsymbol{h}_{j}\right\}_{j=-\infty}^{k-1}\right) ; \boldsymbol{y}_{k} \mid \boldsymbol{h}_{k}, \boldsymbol{x}_{k}\right) \\
& =I\left(\mathbb{E}\left(\boldsymbol{h}_{k} \mid\left\{\boldsymbol{h}_{j}\right\}_{j=-\infty}^{k-1}\right) ; \boldsymbol{h}_{k}\right) \\
& =-\log \sigma_{k}(\infty)
\end{aligned}
$$

where (32) follows from the fact that $\mathbb{E}\left(\boldsymbol{h}_{k} \mid\left\{\boldsymbol{h}_{j}\right\}_{j=-\infty}^{k-1}\right) \rightarrow\left(\boldsymbol{h}_{k}, \boldsymbol{x}_{k}\right) \rightarrow \boldsymbol{y}_{k}$ form a Markov chain. Therefore, we get

$$
\begin{aligned}
\limsup _{\mathrm{SNR} \rightarrow \infty}[C(\mathrm{SNR})-\log \log \mathrm{SNR}] & \leq-1-\gamma-\frac{1}{T} \sum_{k=1}^{T} \log \sigma_{k}(0) \\
& =-1-\gamma-\frac{1}{T} \log \operatorname{det}[\Sigma(\infty)] .
\end{aligned}
$$

The desired result follows by combining (30) and 33.

\section{APPENDIX V}

\section{Proof of Proposition 1}

At high SNR, we have

$$
\begin{aligned}
\left.\int_{-\pi}^{\pi} \log \left[s_{\theta}\left(e^{j \omega}\right)+\frac{4}{\mathrm{SNR}}\right] \mathrm{d} \omega\right|_{\theta=\mathrm{SNR}^{r}}= & \alpha \log \left(\frac{4}{\mathrm{SNR}}\right)+\left(2 \pi-\alpha-\mathrm{SNR}^{-r}\right) \log \left(\mathrm{SNR}^{-r}+\frac{4}{\mathrm{SNR}}\right) \\
& +\mathrm{SNR}^{-r} \log \left(\frac{2 \pi \mathrm{SNR}^{2 r}-2 \pi \mathrm{SNR}^{r}+\alpha \mathrm{SNR}^{r}+1}{\mathrm{SNR}^{r}}+\frac{4}{\mathrm{SNR}}\right) \\
= & -2 \pi \kappa \log \mathrm{SNR}+c(r)+o(1)
\end{aligned}
$$

and thus

$$
\left.\operatorname{var}\left(\boldsymbol{h}_{1} \mid\left\{\boldsymbol{h}_{k}+\frac{2}{\sqrt{\mathrm{SNR}}} \boldsymbol{z}_{1}\right\}_{k=-\infty}^{0}\right)\right|_{s_{\theta}\left(e^{j \omega}\right), \theta=\mathrm{SNR}^{r}}=\frac{e^{\frac{c(r)}{2 \pi}}}{\mathrm{SNR}^{\kappa}}-\frac{4}{\mathrm{SNR}^{2}}+o\left(\frac{1}{\mathrm{SNR}^{\kappa}}\right)
$$

where $\kappa=\frac{\alpha+\min (r, 1)(2 \pi-\alpha)}{2 \pi}$, and

$$
c(r)= \begin{cases}\alpha \log 4 & r \in(0,1) \\ \alpha \log 4+(2 \pi-\alpha) \log 5 & r=1 \\ 2 \pi \log 4 & r \in(1, \infty) .\end{cases}
$$


In the lower bound (14), let $\boldsymbol{x}_{1}$ be uniformly distributed over the set $\left\{z \in \mathbb{C}: \frac{\sqrt{\mathrm{SNR}}}{2} \leq|z| \leq \sqrt{\mathrm{SNR}}\right\}$. By Lemma 2 and Equation [34, we get

$$
\begin{aligned}
C(\mathrm{SNR}) & \geq I\left(\boldsymbol{x}_{1} ; \boldsymbol{h}_{1} \boldsymbol{x}_{1}+\boldsymbol{z}_{1} \mid \mathbb{E}\left(\boldsymbol{h}_{1} \mid\left\{\boldsymbol{h}_{k}+\frac{2}{\sqrt{\mathrm{SNR}}} \boldsymbol{z}_{1}\right\}_{k=-\infty}^{0}\right)\right) \\
& \geq-\log \left[\frac{e^{\frac{c(r)}{2 \pi}}}{\mathrm{SNR}^{\kappa}}-\frac{4}{\mathrm{SNR}}+\frac{8}{5 \mathrm{SNR}}+o\left(\frac{1}{\mathrm{SNR}^{\kappa}}\right)\right]+\log \left(1-\frac{e^{\frac{c(r)}{2 \pi}}}{\mathrm{SNR}}+\frac{4}{\mathrm{SNR}}-o\left(\frac{1}{\mathrm{SNR} \kappa}\right)\right)-\gamma-\log \frac{5 e}{6} \\
& =\kappa \log \mathrm{SNR}+o(\log \mathrm{SNR}) .
\end{aligned}
$$

Therefore,

$$
\liminf _{\mathrm{SNR} \rightarrow \infty, \theta=\mathrm{SNR}^{r}} \frac{\left.C(\mathrm{SNR})\right|_{s_{\theta}\left(e^{j \omega}\right)}}{\log \mathrm{SNR}} \geq \kappa=\frac{\alpha+\min (r, 1)(2 \pi-\alpha)}{2 \pi} .
$$

When $r \geq 1$, we have

$$
\liminf _{\mathrm{SNR} \rightarrow \infty, \theta=\mathrm{SNR}^{r}} \frac{\left.C(\mathrm{SNR})\right|_{s_{\theta}\left(e^{j \omega}\right)}}{\log \mathrm{SNR}} \geq 1 .
$$

Since the noncoherent channel capacity with peak power constraint $|\boldsymbol{x}|^{2} \leq$ SNR is upper bounded by the coherent channel capacity with average power constraint $\mathbb{E}|\boldsymbol{x}|^{2} \leq \mathrm{SNR}$, it follows that

$$
\left.C(\mathrm{SNR})\right|_{s_{\theta}\left(e^{j \omega}\right)} \leq \mathbb{E}_{\boldsymbol{h}} \log \left(1+\mathrm{SNR}|\boldsymbol{h}|^{2}\right) \quad \text { for all } \theta .
$$

where $\boldsymbol{h} \sim \mathcal{C N}(0,1)$. Therefore,

$$
\limsup _{\mathrm{SNR} \rightarrow \infty, \theta=\mathrm{SNR}^{r}} \frac{\left.C(\mathrm{SNR})\right|_{s_{\theta}\left(e^{j \omega}\right)}}{\log \mathrm{SNR}} \leq \lim _{\mathrm{SNR} \rightarrow \infty} \frac{\mathbb{E}_{\boldsymbol{h}} \log \left(1+\mathrm{SNR}|\boldsymbol{h}|^{2}\right)}{\log \mathrm{SNR}}=1 .
$$

The proof is complete.

\section{APPENDIX VI}

\section{EXAMPLE 4}

In the lower bound (14, let $\boldsymbol{x}_{1}$ be uniformly distributed over the set $\left\{z \in \mathbb{C}: \frac{\sqrt{\mathrm{SNR}}}{2} \leq|z| \leq \sqrt{\mathrm{SNR}}\right\}$. By Lemma 2] we get

$$
\begin{aligned}
C(\mathrm{SNR}) \geq & I\left(\boldsymbol{x}_{1} ; \boldsymbol{h}_{1} \boldsymbol{x}_{1}+\boldsymbol{z}_{1} \mid \mathbb{E}\left(\boldsymbol{h}_{1} \mid\left\{\boldsymbol{h}_{k}+\frac{2}{\sqrt{\mathrm{SNR}}} \boldsymbol{z}_{1}\right\}_{k=-\infty}^{0}\right)\right) \\
\geq & -\log \left(\operatorname{var}\left(\boldsymbol{h}_{1} \mid\left\{\boldsymbol{h}_{k}+\frac{2}{\sqrt{\mathrm{SNR}}} \boldsymbol{z}_{1}\right\}_{k=-\infty}^{0}\right)+\frac{8}{5 \mathrm{SNR}}\right) \\
& +\log \left(1-\operatorname{var}\left(\boldsymbol{h}_{1} \mid\left\{\boldsymbol{h}_{k}+\frac{2}{\sqrt{\mathrm{SNR}}} \boldsymbol{z}_{1}\right\}_{k=-\infty}^{0}\right)\right)-\gamma-\log \frac{5 e}{6}
\end{aligned}
$$

where

$$
\begin{aligned}
& \operatorname{var}\left(\boldsymbol{h}_{1} \mid\left\{\boldsymbol{h}_{k}+\frac{2}{\sqrt{\mathrm{SNR}}} \boldsymbol{z}_{1}\right\}_{k=-\infty}^{0}\right) \\
= & \left(\epsilon_{1}+\frac{4}{\mathrm{SNR}}\right)^{\alpha_{1}}\left(\epsilon_{2}+\frac{4}{\mathrm{SNR}}\right)^{\alpha_{2}-\alpha_{1}}\left[\frac{1-\alpha_{1} \epsilon_{1}-\left(\alpha_{2}-\alpha_{1}\right) \epsilon_{2}}{1-\alpha_{2}}+\frac{4}{\mathrm{SNR}}\right]^{1-\alpha_{2}}-\frac{4}{\mathrm{SNR}}
\end{aligned}
$$


By specializing the upper-bound $(8)$ to the case where $T=1$, we obtain

$$
\begin{aligned}
& C(\mathrm{SNR}) \leq \sup _{\boldsymbol{P}_{\boldsymbol{x}_{1}} \in \mathcal{P}_{1}(\mathrm{SNR})} I\left(\boldsymbol{x}_{1}, \mathbb{E}\left(\boldsymbol{h}_{1} \mid\left\{\boldsymbol{h}_{t}+\frac{1}{\sqrt{\mathrm{SNR}}} \boldsymbol{z}_{t}\right\}_{t=-\infty}^{0}\right) ; \boldsymbol{y}_{1}\right) \\
& \leq \sup _{P_{\boldsymbol{x}_{1}} \in \mathcal{P}_{1}(\mathrm{SNR})} I\left(\boldsymbol{x}_{1} ; \boldsymbol{y}_{1}\right)+\sup _{P_{\boldsymbol{x}_{1}} \in \mathcal{P}_{1}(\mathrm{SNR})} I\left(\mathbb{E}\left(\boldsymbol{h}_{1} \mid\left\{\boldsymbol{h}_{t}+\frac{1}{\sqrt{\mathrm{SNR}}} \boldsymbol{z}_{t}\right\}_{t=-\infty}^{0}\right) ; \boldsymbol{y}_{1} \mid \boldsymbol{x}_{1}\right) \\
& \leq \sup _{P_{\boldsymbol{x}_{1}} \in \mathcal{P}_{1}(\mathrm{SNR})} I\left(\boldsymbol{x}_{1} ; \boldsymbol{y}_{1}\right)+\sup _{P_{\boldsymbol{x}_{1}} \in \mathcal{P}_{1}(\mathrm{SNR})} I\left(\mathbb{E}\left(\boldsymbol{h}_{1} \mid\left\{\boldsymbol{h}_{t}+\frac{1}{\sqrt{\mathrm{SNR}}} \boldsymbol{z}_{t}\right\}_{t=-\infty}^{0}\right) ; \boldsymbol{h}_{1}, \boldsymbol{y}_{1} \mid \boldsymbol{x}_{1}\right) \\
& =\sup _{P_{\boldsymbol{x}_{1}} \in \mathcal{P}_{1}(\mathrm{SNR})} I\left(\boldsymbol{x}_{1} ; \boldsymbol{y}_{1}\right)+I\left(\mathbb{E}\left(\boldsymbol{h}_{1} \mid\left\{\boldsymbol{h}_{t}+\frac{1}{\sqrt{\mathrm{SNR}}} \boldsymbol{z}_{t}\right\}_{t=-\infty}^{0}\right) ; \boldsymbol{h}_{1}\right)
\end{aligned}
$$

where

$$
\begin{aligned}
& I\left(\mathbb{E}\left(\boldsymbol{h}_{1} \mid\left\{\boldsymbol{h}_{t}+\frac{1}{\sqrt{\mathrm{SNR}}} \boldsymbol{z}_{t}\right\}_{t=-\infty}^{0}\right) ; \boldsymbol{h}_{1}\right) \\
= & -\log \left[\operatorname{var}\left(\boldsymbol{h}_{1} \mid\left\{\boldsymbol{h}_{t}+\frac{1}{\sqrt{\mathrm{SNR}}} \boldsymbol{z}_{t}\right\}_{t=-\infty}^{0}\right)\right] \\
= & -\log \left\{\left(\epsilon_{1}+\frac{1}{\mathrm{SNR}}\right)^{\alpha_{1}}\left(\epsilon_{2}+\frac{1}{\mathrm{SNR}}\right)^{\alpha_{2}-\alpha_{1}}\left[\frac{1-\alpha_{1} \epsilon_{1}-\left(\alpha_{2}-\alpha_{1}\right) \epsilon_{2}}{1-\alpha_{2}}+\frac{1}{\mathrm{SNR}}\right]^{1-\alpha_{2}}-\frac{1}{\mathrm{SNR}}\right\} .
\end{aligned}
$$

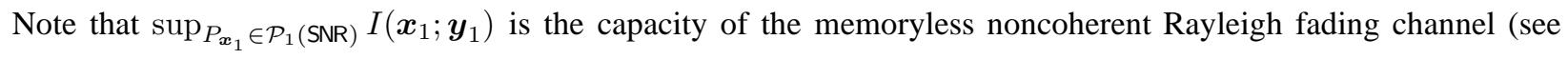
[3] for a nonasymptotic upper bound), and we have $\sup _{P_{\boldsymbol{x}_{1}} \in \mathcal{P}_{1} \text { (SNR) }} I\left(\boldsymbol{x}_{1} ; \boldsymbol{y}_{1}\right) \ll \log$ SNR for large SNR.

By (35) and 36, it is not hard to verify that $\frac{C(\mathrm{SNR})}{\log \mathrm{SNR}}$ is approximately equal to $\alpha_{2}$ for $1 \ll \mathrm{SNR} \leq \frac{1}{\epsilon_{2}}$, and is approximately equal to $\alpha_{1}$ for $\log \frac{1}{\epsilon_{2}} \ll \log \operatorname{SNR} \leq \log \frac{1}{\epsilon_{1}}$.

\section{APPENDIX VII}

\section{AWGN CHANNEL}

By the random coding bound [20], we have

$$
P_{e} \leq e^{-n E_{r}(R)}
$$

where

$E_{r}(R)=\frac{\operatorname{SNR}}{2 e^{R}}\left[e^{R}+1-\left(e^{R}-1\right) \sqrt{1+\frac{4 e^{R}}{\operatorname{SNR}\left(e^{R}-1\right)}}\right]+\log \left\{e^{R}-\frac{\operatorname{SNR}\left(e^{R}-1\right)}{2}\left[\sqrt{1+\frac{4 e^{R}}{\operatorname{SNR}\left(e^{R}-1\right)}}-1\right]\right\}$

if $\log \left[\frac{1}{2}+\frac{\mathrm{SNR}}{4}+\frac{1}{2} \sqrt{1+\frac{\mathrm{SNR}^{2}}{4}}\right] \leq R \leq \log (1+\mathrm{SNR})$, and

$E_{r}(R)=1+\frac{\mathrm{SNR}}{2}-\sqrt{1+\frac{\mathrm{SNR}^{2}}{4}}+\log \left(\frac{1}{2}-\frac{\mathrm{SNR}}{4}+\frac{1}{2} \sqrt{1+\frac{\mathrm{SNR}^{2}}{4}}\right)+\log \left(\frac{1}{2}+\frac{\mathrm{SNR}}{4}+\frac{1}{2} \sqrt{1+\frac{\mathrm{SNR}^{2}}{4}}\right)-R$

if $R<\log \left[\frac{1}{2}+\frac{\mathrm{SNR}}{4}+\frac{1}{2} \sqrt{1+\frac{\mathrm{SNR}^{2}}{4}}\right]$. 
Let $R(\mathrm{SNR})=\log \mathrm{SNR}-\log \eta$ where $\eta \in(1,2)$. By 37,

$$
\begin{aligned}
& \lim _{\mathrm{SNR} \rightarrow \infty} E_{r}(R(\mathrm{SNR})) \\
= & \lim _{\mathrm{SNR} \rightarrow \infty} \frac{\eta}{2}\left[\frac{\mathrm{SNR}}{\eta}+1-\left(\frac{\mathrm{SNR}}{\eta}-1\right) \sqrt{1+\frac{4}{\mathrm{SNR}-\eta}}\right]+\log \left\{\frac{\mathrm{SNR}}{\eta}-\frac{\mathrm{SNR}(\mathrm{SNR}-\eta)}{2 \eta}\left[\sqrt{1+\frac{4}{\mathrm{SNR}-\eta}}-1\right]\right\} \\
= & \lim _{\mathrm{SNR} \rightarrow \infty} \frac{\eta}{2}\left[\frac{\mathrm{SNR}}{\eta}+1-\frac{\mathrm{SNR}-\eta}{\eta}\left(1+\frac{2}{\mathrm{SNR}-\eta}\right)\right]+\log \left\{\frac{\mathrm{SNR}}{\eta}-\frac{\mathrm{SNR}(\mathrm{SNR}-\eta)}{2 \eta}\left[\frac{2}{\mathrm{SNR}-\eta}-\frac{2}{(\mathrm{SNR}-\eta)^{2}}\right]\right\} \\
= & \eta-1-\log \eta \\
>0 & .
\end{aligned}
$$

For any $P_{e}>0$, we can find an $n$ such that

$$
e^{-n(\eta-1-\log \eta)}<P_{e}
$$

Therefore, for any $P_{e}>0$, there exist a sequence of codebooks with rate $R(\mathrm{SNR})$ and fixed codeword length $n$ such that

$$
\lim _{\mathrm{SNR} \rightarrow \infty} \frac{R(\mathrm{SNR})}{\log \mathrm{SNR}}=1
$$

and $\lim \sup _{\mathrm{SNR} \rightarrow \infty} P_{e}(\mathrm{SNR}) \leq P_{e}$.

\section{APPENDIX VIII \\ Coherent Rayleigh FAding Channel}

It was shown in [21] that

$$
E_{r}(R)=\max _{0 \leq \rho \leq 1}\left[-\log \mathbb{E}_{\boldsymbol{h}}\left(1+\frac{\mathrm{SNR}}{1+\rho}|\boldsymbol{h}|^{2}\right)^{-\rho}-\rho R\right]
$$

where $\boldsymbol{h} \sim \mathcal{C N}(0,1)$.

Choosing $R(\mathrm{SNR})=\log \mathrm{SNR}-\log \log \mathrm{SNR}-c$ and $\rho=1$, we get

$$
\begin{aligned}
\liminf _{\mathrm{SNR} \rightarrow \infty} E_{r}(R(\mathrm{SNR})) & \geq \liminf _{\mathrm{SNR} \rightarrow \infty}\left[-\log \mathbb{E}_{\boldsymbol{h}}\left(1+\frac{\mathrm{SNR}}{1+\rho}|\boldsymbol{h}|^{2}\right)^{-1}-\log \mathrm{SNR}+\log \log \mathrm{SNR}+c\right] \\
& =\liminf _{\mathrm{SNR} \rightarrow \infty}\left[-\log \mathbb{E}_{\boldsymbol{h}}\left(\frac{1}{\mathrm{SNR}}+\frac{1}{2}|\boldsymbol{h}|^{2}\right)^{-1}+\log \log \mathrm{SNR}+c\right] \\
& =\liminf _{\mathrm{SNR} \rightarrow \infty}\left\{-\log \left[\int_{0}^{\infty}\left(\frac{1}{\mathrm{SNR}}+\frac{t}{2}\right)^{-1} e^{-t} \mathrm{~d} t\right]+\log \log \mathrm{SNR}+c\right\} \\
& =\liminf _{\mathrm{SNR} \rightarrow \infty}\left\{-\log \left[\int_{0}^{1}\left(\frac{1}{\mathrm{SNR}}+\frac{t}{2}\right)^{-1} e^{-t} \mathrm{~d} t+\int_{1}^{\infty}\left(\frac{1}{\mathrm{SNR}}+\frac{t}{2}\right)^{-1} e^{-t} \mathrm{~d} t\right]+\log \log \mathrm{SNR}+c\right\} \\
& \geq \liminf _{\mathrm{SNR} \rightarrow \infty}\left\{-\log \left[\int_{0}^{1}\left(\frac{1}{\mathrm{SNR}}+\frac{t}{2}\right)^{-1} \mathrm{~d} t+\int_{1}^{\infty} 2 e^{-t} \mathrm{~d} t\right]+\log \log \mathrm{SNR}+c\right\} \\
& =\liminf _{\mathrm{SNR} \rightarrow \infty}\left[-\log \left[2 \log \left(2+2 \mathrm{SNR}+\mathrm{SNR}^{2}\right)-2 \log (2+2 \mathrm{SNR})+2 e^{-1}\right]+\log \log \mathrm{SNR}+c\right] \\
& =-\log 2+c
\end{aligned}
$$


which is positive if $c>\log 2$.

Therefore, for any $P_{e}>0$, we can find a sequence of codebooks with rate $R(\mathrm{SNR})$ and fixed codeword length $n$ such that

$$
\lim _{\mathrm{SNR} \rightarrow \infty} \frac{R(\mathrm{SNR})}{\log \mathrm{SNR}}=1
$$

and $\lim \sup _{\mathrm{SNR} \rightarrow \infty} P_{e}(\mathrm{SNR}) \leq P_{e}$

\section{APPENDIX IX}

EXAMPLE 7

We can compute that

$$
\begin{aligned}
& \Psi(\{1\}, \mathrm{SNR})=\Psi(\{2\}, \mathrm{SNR})=\Psi(\{3\}, \mathrm{SNR})=2 \pi \log (1+\mathrm{SNR}) \\
& \Psi(\{1,3\}, \mathrm{SNR})=\Psi(\{2,3\}, \mathrm{SNR})=\pi \log \left(1+2 \mathrm{SNR}+\mathrm{SNR}^{2}-|\rho|^{2} \mathrm{SNR}\right) \\
& \Psi(\{1,2\}, \mathrm{SNR})=\pi \log (1+2 \mathrm{SNR}) \\
& \Psi(\{1,2,3\}, \mathrm{SNR})=\frac{2 \pi}{3} \log \left(1+3 \mathrm{SNR}+2 \mathrm{SNR}^{2}-2|\rho|^{2} \mathrm{SNR}^{2}\right) .
\end{aligned}
$$

It can be verified that

$$
\Psi(\{1\}, \mathrm{SNR})=\Psi(\{2\}, \mathrm{SNR})=\Psi(\{3\}, \mathrm{SNR}) \geq \Psi(\{1,3\}, \mathrm{SNR})=\Psi(\{2,3\}, \mathrm{SNR}) \geq \Psi(\{1,2\}, \mathrm{SNR}) .
$$

So the optimal $\mathcal{M}^{*}$ is either $\{1,2\}$ or $\{1,2,3\}$. Setting $\Psi(\{1,2\}$, SNR $)=\Psi(\{1,2,3\}$, SNR $)$ yields

$$
\left(1+3 \mathrm{SNR}+2 \mathrm{SNR}^{2}-2|\rho|^{2} \mathrm{SNR}^{2}\right)^{2}=(1+2 \mathrm{SNR})^{3}
$$

which, after some algebraic manipulation, is equivalent to

$$
1-4|\rho|^{2}+\left(4-12|\rho|^{2}\right) \operatorname{SNR}+\left(4-8|\rho|^{2}+4|\rho|^{4}\right) \mathrm{SNR}^{2}=0 .
$$

The above equation has two solutions

$$
\mathrm{SNR}_{1}=\frac{-2|\rho|-1}{2(1+|\rho|)^{2}}, \quad \mathrm{SNR}_{2}=\frac{2|\rho|-1}{2(1-|\rho|)^{2}} .
$$

$\mathrm{SNR}_{1}$ can be discarded since it is always negative. $\mathrm{SNR}_{2}$ is positive for $|\rho| \in\left(\frac{1}{2}, 1\right)$. When $|\rho| \in\left(\frac{1}{2}, 1\right)$, it can be verified that $\Psi(\{1,2\}, \mathrm{SNR})>\Psi(\{1,2,3\}$, SNR $)$ if SNR $<\mathrm{SNR}_{2}$, and $\Psi(\{1,2\}$, SNR $)<\Psi(\{1,2,3\}$, SNR $)$ if $\mathrm{SNR}>\mathrm{SNR}_{2}$. When $|\rho| \in\left[0, \frac{1}{2}\right], \mathrm{SNR}_{2}$ is non-positive. In this case, we have $\Psi(\{1,2\}, \mathrm{SNR})>\Psi(\{1,2,3\}, \mathrm{SNR})$ for all $S N R>0$.

\section{REFERENCES}

[1] T. Marzetta and B. Hochwald, "Capacity of a mobile multiple-antenna communication link in Rayleigh flat fading," IEEE Trans. Inform. Theory, vol. 45, pp. 139-157, Jan. 1999.

[2] L. Zheng and D. N. C. Tse, "Communication on the Grassmann manifold: A geometric approach to the noncoherent multiple-antenna channel," IEEE Trans. Inform. Theory, vol. 48, pp. 359-383, Feb. 2002. 
[3] A. Lapidoth and S. Moser, "Capacity bounds via duality with applications to multi-antenna systems on flat fading channels," IEEE Trans. Inform. Theory, vol. 49, pp. 2426-2467, Oct. 2003.

[4] A. Lapidoth and S. Moser, "The fading number of single-input multiple-output fading channels with memory," IEEE Trans. Inform. Theory, vol. 52, pp. 437-453, Feb. 2006.

[5] T. Koch and A. Lapidoth, "The fading number and degrees of freedom in non-coherent MIMO fading channels: a peace pipe," Proceedings 2005 IEEE International Symposium on Information Theory (ISIT), Adelaide, Australia, Sept. 4-9, 2005

[6] A. Lapidoth, "On the asymptotic capacity of stationary Gaussian fading channels," IEEE Trans. Inform. Theory, vol. 51, pp. 437-446, Feb. 2005.

[7] Y. Liang and V. V. Veeravalli, "Capacity of noncoherent time-selective Rayleigh-fading channels," IEEE Trans. Inform. Theory, vol. 50, pp. 3095-3110, Dec. 2004.

[8] Yu. A. Rozanov, Stationary Random Processes. San Francisco, CA: Holden-Day, 1967.

[9] S. Verdú, "On channel capacity per unit cost," IEEE Trans. Inform. Theory, vol. 36, pp. 1019-1030, Sept. 1990.

[10] R. Etkin and D. Tse, "Degrees of freedom in some underspread MIMO fading channels," IEEE Trans. Inform. Theory, vol. 52, pp. 15761608, Apr. 2006.

[11] L. Zheng and D. N. C. Tse, "Diversity and multiplexing: A fundamental tradeoff in multiple antenna channels," IEEE Trans. Inform. Theory, vol. 49, pp. 1073-1096, May 2003.

[12] G. M. Goluzin, The Geometrical Theory of Functions of a Complex Variable. Moscow: Nauka, 1966.

[13] N. M. Babayan, "On asymptotic behavior of the prediction error in the singular case," Theory of Probability and Its Applications, vol. 29, no. 1, pp. 147-150, 1985.

[14] V. Sethuraman and B. Hajek, "Capacity per unit energy of fading channels with a peak constraint," IEEE Trans. Inform. Theory, vol. 51, pp. 3102-3120, Sept. 2005.

[15] S. Boyd and L. Vandenberghe, Convex Optimization. Cambridge, U.K.: Cambridge Univ. Press, 2004.

[16] R. Horn and C. Johnson, Matrix Analysis. Cambridge, U.K.: Cambridge Univ. Press, 1985.

[17] S. Verdú, "Spectral efficiency in the wideband regime," IEEE Trans. Inform. Theory, vol. 48, pp. 1319-1343, Feb. 2002.

[18] L. Zheng, D.Tse, and M. Médard, "Channel coherence in the low SNR regime," IEEE Trans. Inform. Theory, submitted for publication.

[19] S. Ray, M. Médard and L. Zheng, "On non-coherent MIMO capacity in the wideband regime: interplay of SNR, antennas and coherence," IEEE Trans. Inform. Theory, submitted for publication.

[20] R. Gallager, Information Theory and Reliable Communication. New York: Wiley, 1968.

[21] İ. E. Telatar, "Capacity of multi-antenna Gaussian channels," Europ. Trans. Telecommun., vol. 10, no. 6, pp. 585-595, 1999. 